\title{
The center of the affine nilTemperley-Lieb algebra
}

\author{
Georgia Benkart $^{1} \cdot$ Joanna Meinel $^{2,3}$
}

Received: 30 June 2015 / Accepted: 12 March 2016 / Published online: 21 April 2016

(C) The Author(s) 2016. This article is published with open access at Springerlink.com

\begin{abstract}
We give a description of the center of the affine nilTemperley-Lieb algebra based on a certain grading of the algebra and on a faithful representation of it on fermionic particle configurations. We present a normal form for monomials, hence construct a basis of the algebra, and use this basis to show that the affine nilTemperley-Lieb algebra is finitely generated over its center. As an application, we obtain a natural embedding of the affine nilTemperley-Lieb algebra on $N$ generators into the affine nilTemperley-Lieb algebra on $N+1$ generators.
\end{abstract}

\section{Introduction}

The main goal of this work is to describe the center of the affine nilTemperley-Lieb algebra $\mathrm{n} \widehat{T L}_{N}$ over any ground field. Only two tools are used: a fine grading on $n \widehat{T L}_{N}$ and a representation of $n \widehat{T L}_{N}$ on fermionic particle configurations on a circle. It is essential that this graphical representation be faithful (see [12, Prop. 9.1]). We provide an alternative proof of that fact by constructing a basis for $\mathrm{nTL}_{N}$ that is especially adapted to the problem. This basis has further advantages: It can be used to prove that the affine nilTemperley-Lieb algebra is

The authors thank Catharina Stroppel for many helpful discussions and express their gratitude to the Mathematical Sciences Research Institute (MSRI) where their joint research began. The authors thank the referee for very detailed remarks. The second author would like to thank Daniel Tubbenhauer for remarks on the embeddings. This work is part of the second author's Ph.D. project at the MPIM Bonn, supported by a grant of the Deutsche Telekom Stiftung.

$凶$ Joanna Meinel

joanna@math.uni-bonn.de

Georgia Benkart

benkart@math.wisc.edu

1 Department of Mathematics, University of Wisconsin-Madison, Madison, WI 53706, USA

2 Mathematical Institute, University of Bonn, 53115 Bonn, Germany

3 Max Planck Institute for Mathematics, Vivatsgasse 7, 53111 Bonn, Germany 
finitely generated over its center. Also, it can be used to exhibit an explicit embedding of $\mathrm{n} \widehat{\mathrm{TL}}_{N}$ into $\widehat{\mathrm{TL}}_{N+1}$ defined on basis elements that otherwise would not be apparent, since the defining relations of these algebras are affine, and there is no embedding of the corresponding Coxeter graphs.

For a ground field $\mathbb{k}$, the affine nilTemperley-Lieb algebra $\widehat{n T L}_{N}$ is the unital associative k-algebra given by $N$ generators $a_{0}, \ldots, a_{N-1}$ and nil relations $a_{i}^{2}=0$ and $a_{i} a_{i \pm 1} a_{i}=0$ for all $i$. Generators that are far apart commute, i.e. $a_{i} a_{j}=a_{j} a_{i}$ for $i-j \neq \pm 1 \bmod N$. In these relations, the indices are interpreted modulo $N$ so that the generators $a_{0}$ and $a_{N-1}$ are neighbours that do not commute. The subalgebra of $\mathrm{nTL}_{N}$ generated by $a_{1}, \ldots, a_{N-1}$ is the (finite) nilTemperley-Lieb algebra $\mathrm{nTL}_{N}$, as in [19]. The affine nilTemperley-Lieb algebra appears in many different settings, which we describe next.

1. $\widehat{\mathrm{nL}}_{N}$ is a quotient of the affine nilCoxeter algebra of type $\tilde{\mathrm{A}}_{N-1}$.

The affine nilCoxeter algebra $\widehat{\mathrm{U}}_{N}$ of type $\tilde{\mathrm{A}}_{N-1}$ over a field $\mathbb{k}$ is the unital associative algebra generated by elements $u_{i}, 0 \leq i \leq N-1$, satisfying the relations $u_{i}^{2}=0$; $u_{i} u_{j}=u_{j} u_{i}$ for $i-j \neq \pm 1 \bmod N$; and $u_{i} u_{i+1} u_{i}=u_{i+1} u_{i} u_{i+1}$ for $1 \leq i \leq N-1$, where the subscripts are read modulo $N$. The algebra $\widehat{n T L}_{N}$ is isomorphic to the quotient of $\widehat{\mathrm{U}}_{N}$ obtained by imposing the additional relations $u_{i} u_{i+1} u_{i}=u_{i+1} u_{i} u_{i+1}=0$ for $1 \leq i \leq N-1$. The affine nilCoxeter algebra is closely connected with affine Schur functions, $k$-Schur functions, and the affine Stanley symmetric functions, which are related to reduced word decompositions in the affine symmetric group (see e.g. $[14,15]$ ). The nilCoxeter algebra $\bigcup_{N}$ has generators $u_{i}, 1 \leq i \leq N-1$, which satisfy the same relations as they do in $\widehat{U}_{N}$. It first appeared in work on the cohomology of flag varieties [3] and has played an essential role in studies on Schubert polynomials, Stanley symmetric functions, and the geometry of flag varieties (see for example $[8,11,16,17]$ ). The definition of $\bigcup_{N}$ was inspired by the divided difference operators $\partial_{i}$ on polynomials in variables $\mathbf{x}=\left\{x_{1}, \ldots, x_{N}\right\}$ defined by

$$
\partial_{i}(f)=\frac{f(\mathbf{x})-f\left(\sigma_{i} \mathbf{x}\right)}{x_{i}-x_{i+1}},
$$

where the transposition $\sigma_{i}$ fixes all the variables except for $x_{i}$ and $x_{i+1}$, which it interchanges. The operators $\partial_{i}$ satisfy the nilCoxeter relations above, and applications of these relations enabled Fomin and Stanley [8] to recover known properties and establish new properties of Schubert polynomials.

The algebra $\bigcup_{N}$ belongs to a two-parameter family of algebras having generators $u_{i}$, $1 \leq i \leq N-1$, which satisfy the relations $u_{i} u_{j}=u_{j} u_{i}$ for $|i-j|>1$ and $u_{i} u_{i+1} u_{i}=$ $u_{i+1} u_{i} u_{i+1}$ for $1 \leq i \leq N-2$ from above, together with the relation $u_{i}^{2}=\alpha u_{i}+\beta$ for all $i$, where $\alpha, \beta$ are fixed parameters. In particular, the specialization $\alpha=\beta=0$ yields the nilCoxeter algebra; $\alpha=0, \beta=1$ gives the standard presentation of the group algebra of the symmetric group $k_{N} S_{N}$; and $\alpha=q-1, \beta=q$ gives the Hecke algebra $\mathrm{H}_{N}(q)$ of type $\mathrm{A}$.

Motivated by categorification results in [6], Khovanov [10] introduced restriction and induction functors $\mathrm{F}_{D}$ and $\mathrm{F}_{X}$ corresponding to the natural inclusion of algebras $\mathrm{U}_{N} \hookrightarrow$ $\bigcup_{N+1}$ on the direct sum $\mathcal{C}$ of the categories $\mathcal{C}_{N}$ of finite-dimensional $\bigcup_{N}$-modules. These functors categorify the Weyl algebra of differential operators with polynomial coefficients in one variable and correspond to the Weyl algebra generators $\partial$ and $x$ (derivative and multiplication by $x$ ), which satisfy the relation $\partial x-x \partial=1$.

Brichard [5] used a diagram calculus on cylinders to determine the dimension of the center of $\bigcup_{N}$ and to describe a basis of the center for which the multiplication is trivial. 
In this diagram calculus on $N$ strands, the generator $u_{i}$ corresponds to a crossing of the strands $i$ and $i+1$. The nil relation $u_{i}^{2}=0$ is represented by demanding that any two strands may cross at most once; otherwise the diagram is identified with zero.

2. $\mathrm{nTL}_{N}$ is a quotient of the negative part of the universal enveloping algebra of the affine Lie algebra $\widehat{\mathfrak{s l}}_{N}$.

The negative part $U^{-}$of the universal enveloping algebra $U$ of the affine Lie algebra $\widehat{\mathfrak{s l}}_{N}$ has generators $f_{i}, 0 \leq i \leq N-1$, which satisfy the Serre relations

$$
\begin{aligned}
& f_{i}^{2} f_{i+1}-2 f_{i} f_{i+1} f_{i}+f_{i+1} f_{i}^{2}=0 \\
& \quad=f_{i+1}^{2} f_{i}-2 f_{i+1} f_{i} f_{i+1}+f_{i} f_{i+1}^{2} \text { and } f_{i} f_{j}=f_{j} f_{i} \text { for } i-j \neq \pm 1 \bmod N
\end{aligned}
$$

(all indices modulo $N$ ). Factoring $U^{-}$by the ideal generated by the elements $f_{i}^{2}, 0 \leq$ $i \leq N-1$, gives $\mathrm{nTL}_{N}$ whenever the characteristic of $\mathbb{k}$ is different from 2 .

3. $\mathrm{n}_{\widehat{T L}_{N}}$ acts on the small quantum cohomology ring of the Grassmannian.

As in [19, Sec. 2], (see also [12]), consider the cohomology $\operatorname{ring} \mathrm{H}^{\bullet}(\mathrm{Gr}(k, N))$ with integer coefficients for the Grassmannian $\operatorname{Gr}(k, N)$ of $k$-dimensional subspaces of $\mathbb{k}^{N}$. It has a basis given by the Schubert classes $\left[\Omega_{\lambda}\right]$, where $\lambda$ runs over all partitions with $k$ parts, the largest part having size $N-k$. By recording the $k$ vertical and $N-k$ horizontal steps that identify the Young diagram of $\lambda$ inside the northwest corner of a $k \times(N-k)$ rectangle, such a partition corresponds to a $(0,1)$-sequence of length $N$ with $k$ ones (resp. $N-k$ zeros) in the positions corresponding to the vertical (respectively horizontal) steps. As a $\mathbb{Z}[q]$-module for an indeterminate $q$, the quantum cohomology ring of the Grassmannian is given by $\mathrm{qH}^{\bullet}(\mathrm{Gr}(k, N))=\mathbb{Z}[q] \otimes_{\mathbb{Z}} \mathrm{H}^{\bullet}(\mathrm{Gr}(k, N))$ together with a $q$-multiplication. The $\widehat{n T L}_{N}$-action can be defined combinatorially on

$$
\mathrm{qH}^{\bullet}(\operatorname{Gr}(k, N)) \cong \operatorname{span}_{\mathbb{Z}[q]}\{(0,1) \text {-sequences of length } N \text { with } k \text { ones }\}
$$

as described in the next item, and the multiplication of two Schubert classes $\left[\Omega_{\lambda}\right] \cdot\left[\Omega_{\mu}\right]$ is equal to $s_{\lambda} \cdot\left[\Omega_{\mu}\right]$ where $s_{\lambda}$ is a certain Schur polynomial in the generators of $\mathrm{nTL}_{N}$ as in [19, Cor. 8.3].

4. $\mathrm{nTL}_{N}$ acts faithfully on fermionic particle configurations on a circle.

This is the graphical representation from [12] (see also [19]), which we use in our description of the center of $\mathrm{nTL}_{N}$. First, a $(0,1)$-sequence with $k$ ones is identified with a circular particle configuration having $N$ positions, where the $k$ particles are distributed at the position on the circle that corresponds to their position in the sequence, so that there is at most one particle at each position. On the space

$\operatorname{span}_{\mathbb{k}[q]}$ \{fermionic particle configurations of $k$ particles on a circle with $N$ positions\},

the generators $a_{i}$ of $\widehat{\mathrm{nL}}_{N}$ act by sending a particle lying at position $i$ to position $i+1$. Additionally, the particle configuration is multiplied by $\pm q$ when applying $a_{0}$. The precise definition is given in Sect. 4, but here is a representative picture (Fig. 1).

5. $\widehat{\mathrm{TL}}_{N}$ appears as a subalgebra of the annihilation/creation algebra.

The finite nilTemperley-Lieb algebra is a subalgebra of the Clifford algebra having generators $\left\{\xi_{i}, \xi_{i}^{*} \mid 0 \leq i \leq N-1\right\}$ and relations $\xi_{i} \xi_{j}+\xi_{j} \xi_{i}=0, \xi_{i}^{*} \xi_{j}^{*}+\xi_{j}^{*} \xi_{i}^{*}=0$, $\xi_{i} \xi_{j}^{*}+\xi_{j}^{*} \xi_{i}=\delta_{i j}$. The Clifford generators $\xi_{i}$ (resp. $\xi_{i}^{*}$ ) act on the fermionic particle configurations by annihilation (resp. creation) of a particle at position $i$. The finite nilTemperley-Lieb algebra appears inside the Clifford algebra via $a_{i} \mapsto \xi_{i+1}^{*} \xi_{i}$. As discussed in [12, Sec. 8], the affine nilTemperley-Lieb algebra is a $q$-deformation of this construction. 

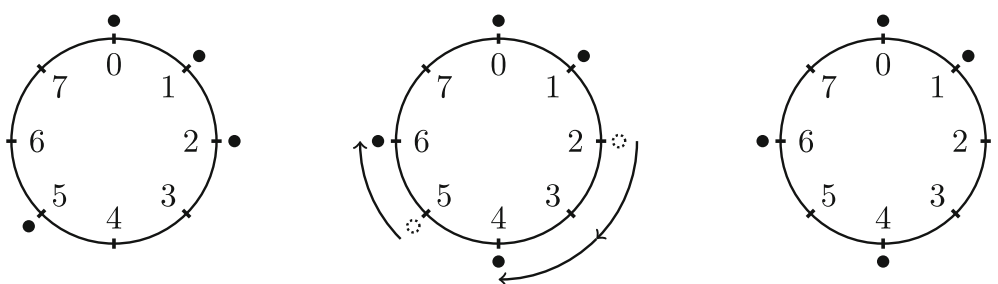

Fig. $1 N=8$ : Application of $a_{3} a_{2} a_{5}$ to the particle configuration $(0,1,2,5)$ gives $(0,1,4,6)$

6. $\widehat{\mathrm{nL}}_{N}$ is the associated graded algebra of the affine Temperley-Lieb algebra.

The affine Temperley-Lieb algebra $\widehat{T L}_{N}(\delta)$ has the usual commuting relations and the relations $a_{i} a_{i \pm 1} a_{i}=a_{i}$ and $a_{i}^{2}=\delta a_{i}$ for some parameter $\delta \in \mathbb{k}$ instead of the nil relations (where again all indices are $\bmod N$ ). It is a filtered algebra with its $\ell$ th filtration space generated by all monomials of length $\leq \ell$. Since its associated graded algebra is $\mathrm{n} \widehat{T L}_{N}$ for any value of $\delta$, elements of $\mathrm{nTL}_{N}$ can be identified with reduced expressions in $\widehat{T L}_{N}(\delta)$.

The diagrammatic structure of $\widehat{\mathrm{TL}}_{N}(\delta)$ is given by the same pictures as for the TemperleyLieb algebra, but now the diagrams are wrapped around the cylinder (see e.g. [7,13]). The top and bottom of the cylinder each have $N$ nodes. Monomials in the affine TemperleyLieb algebra are represented by diagrams of $N$ non-crossing strands, each connecting a pair of those $2 N$ nodes. Multiplication of two monomials is realized by stacking the cylinders one on top of the other, and connecting and smoothing the strands. Whenever the strands form a circle, this is removed from the diagram at the expense of multiplying by the parameter $\delta$. The relation $a_{i} a_{i \pm 1} a_{i}=a_{i}$ corresponds to the isotopy between a strand that changes direction and a strand that is pulled straight.

In contrast, this diagrammatic realization for the affine nilTemperley-Lieb algebra would not respect isotopy: The relation $a_{i} a_{i \pm 1} a_{i}=0$ implies that strands which change the direction are identified with zero. Nevertheless, the diagram of a reduced expression in $\widehat{\mathrm{TL}}_{N}$ may be considered as an element of $n \widehat{\mathrm{TL}}_{N}$. Such a diagram consists of a number (possibly 0) of arcs that connect two nodes on the top of the cylinder, the same number of arcs connecting two nodes on the bottom, and arcs that connect a top node and a bottom one. The latter arcs wrap around the cylinder either all in a strictly clockwise direction or all in a strictly counterclockwise way. Since the multiplication of two such diagrams may give zero, we will not use this diagrammatic realization here.

We proceed as follows: In Sect. 2, we introduce the notation used in this article. The $\mathbb{Z}^{N}$-grading of $\widehat{n T L}_{N}$ is given is Sect. 3, and its importance for the description of the center is discussed. In Sect. 4, we give a detailed definition of the $\widehat{n T L}_{N}$-action on particle configurations on a circle. We also define special monomials that serve as the projections onto a single particle configuration (up to multiplication by $\pm q$ ). Theorem 4.5 of that section recalls [12, Prop. 9.1] stating that the representation is faithful. In [12], this fact is deduced from the finite nilTemperley-Lieb algebra case, as treated in [4] and [2, Prop. 2.4.1]. We give a complete, self-contained proof in Sect. 8. Our proof is elementary and relies on the construction of a basis. Section 5 contains the main result (Theorem 5.5) of this article:

Theorem The center of $n \widehat{T L}_{N}$ is the subalgebra

$$
\mathrm{C}_{N}=\operatorname{Cent}\left(n \widehat{T L}_{N}\right)=\left\langle 1, t_{1}, \ldots, t_{N-1}\right\rangle \cong \frac{\mathbb{k}\left[t_{1}, \ldots, t_{N-1}\right]}{\left(t_{k} t_{\ell} \mid k \neq \ell\right)},
$$


where the generator $\boldsymbol{t}_{k}=(-1)^{k-1} \sum_{|\mathbf{I}|=k} a(\hat{\mathbf{I}})$ is the sum of monomials $a(\hat{\mathbf{I}})$ corresponding to particle configurations given by increasing sequences $\mathbf{I}=\left\{1 \leq i_{1}<\cdots<i_{k} \leq N\right\}$ of length $k$. The monomial $a(\hat{\mathbf{I}})$ sends particle configurations with $n \neq k$ particles to 0 and acts on a particle configuration with $k$ particles by projecting onto I and multiplying by $(-1)^{k-1} q$. Hence, $\boldsymbol{t}_{k}$ acts as multiplication by $q$ on the configurations with $k$ particles.

Our $N-1$ central generators $\mathbf{t}_{k}$ are essentially the $N-1$ central elements constructed by Postnikov. Lemma 9.4 of [19] gives an alternative description of $\mathbf{t}_{k}$ as product of the $k$ th elementary symmetric polynomial (with factors cyclically ordered) with the $(N-k)$ th complete homogeneous symmetric polynomial (with factors reverse cyclically ordered) in the noncommuting generators of $\mathrm{nTL}_{N}$. The above theorem shows that in fact these elements generate the entire center of $n \widehat{T L}_{N}$. In Sect. 6, we establish that $n \widehat{T L}_{N}$ is finitely generated over its center. In Sect. 7, we define a monomial basis for $\mathrm{nTL}_{N}$ indexed by pairs of particle configurations together with a natural number indicating how often the particles have been moved around the circle. A proof that this is indeed a basis of $\mathrm{nTL}_{N}$ can be found in Sect. 8 . With this basis at hand, we obtain inclusions $\mathrm{nTL}_{N} \subset \mathrm{nTL} \widehat{T L}_{N+1}$. The inclusions are not as obvious as those for the nilCoxeter algebra $\bigcup_{N}$ having underlying Coxeter graph of type $\mathrm{A}_{N-1}$, since one cannot deduce them from embeddings of the affine Coxeter graphs. Our result, Theorem 7.1, reads as follows:

Theorem For all $0 \leq m \leq N-1$, there are unital algebra embeddings $\varepsilon_{m}: n \widehat{T L}_{N} \rightarrow$ $n \widehat{T L}_{N+1}$ given by

$$
a_{i} \mapsto a_{i} \text { for } 0 \leq i \leq m-1, \quad a_{m} \mapsto a_{m+1} a_{m}, \quad a_{i} \mapsto a_{i+1} \text { for } m+1 \leq i \leq N-1 .
$$

In Sect. 8, we show how to construct the monomial basis, namely by using a normal form algorithm that reorders the factors of a nonzero monomial. Our basis is reminiscent of the Jones normal form for reduced expressions of monomials in the Temperley-Lieb algebra, as discussed in [20], and is characterised in Theorem 8.6 as follows: (See also Theorem 7.5 which gives a different description.)

Theorem (Normal form) Every nonzero monomial in the generators $a_{j}$ of $n \widehat{T L}_{N}$ can be rewritten uniquely in the form

$$
\left(a_{i_{1}}^{(m)} \ldots a_{i_{k}}^{(m)}\right) \ldots\left(a_{i_{1}}^{(n+1)} \ldots a_{i_{k}}^{(n+1)}\right)\left(a_{i_{1}}^{(n)} \ldots a_{i_{k}}^{(n)}\right) \ldots\left(a_{i_{1}}^{(1)} \ldots a_{i_{k}}^{(1)}\right)\left(a_{i_{1}} \ldots a_{i_{k}}\right)
$$

with $a_{i_{\ell}}^{(n)} \in\left\{1, a_{0}, a_{1}, \ldots, a_{N-1}\right\}$ for all $1 \leq n \leq m, 1 \leq \ell \leq k$, such that

$$
a_{i_{\ell}}^{(n+1)} \in\left\{\begin{array}{lll}
\{1\} & \text { if } & a_{i_{\ell}}^{(n)}=1, \\
\left\{1, a_{j+1}\right\} & \text { if } & a_{i_{\ell}}^{(n)}=a_{j} .
\end{array}\right.
$$

The factors $a_{i_{1}}, \ldots, a_{i_{k}}$ are determined by the property that the generator $a_{i_{\ell}-1}$ does not appear to the right of $a_{i_{\ell}}$ in the original presentation of the monomial. Alternatively, every nonzero monomial is uniquely determined by the following data from its action on the graphical representation:

- the input particle configuration with the minimal number of particles on which it acts nontrivially,

- the output particle configuration,

- the power of $q$ by which it acts. 
For the proof of this result, we recall a characterisation of the nonzero monomials in $\widehat{n T L}_{N}$ from [9]. Then we prove faithfulness of the graphical representation of $\mathrm{nTL}_{N}$ by describing explicitly the matrices representing our basis elements. Al Harbat [1] has recently described a normal form for fully commutative elements of the affine Temperley-Lieb algebra, which gives a different normal form when passing to $\widehat{n T L}_{N}$.

Our results hold over an arbitrary ground field $\mathbb{k}$, even one of characteristic 2 , simply by ignoring signs in that case. In fact, our arguments work for any associative commutative unital ground ring $R$ by replacing $\mathbb{k}$-vector spaces and $\mathbb{k}$-algebras with free $R$-modules and $R$ algebras, respectively. In particular, the affine nilTemperley-Lieb algebra over $\mathbb{k}$ is replaced by the $R$-algebra with the same generators and relations, and the polynomial ring $\mathbb{k}[q]$ is replaced by $R[q]$. We can even drop the assumption that the ring $R$ is commutative if we slightly modify the statements about the center. This is possible because our arguments mainly rely on investigating monomials in the generators of $\mathrm{nTL}_{N}$. However, for simplicity we have chosen to assume $\mathbb{k}$ is a field throughout the article.

\section{Notation}

Let $\mathbb{k}$ be any field, and assume $N$ is a positive integer. The affine nilTemperley-Lieb algebra $\mathrm{nTL}_{N}$ of rank $N$ is the unital associative $\mathbb{k}$-algebra generated by elements $a_{0}, \ldots, a_{N-1}$ subject to the defining relations

$$
\begin{aligned}
a_{i}^{2} & =0 & & \text { for all } 0 \leq i \leq N-1, \\
a_{i} a_{j} & =a_{j} a_{i} & & \text { for all } i-j \neq \pm 1 \bmod \\
a_{i} a_{i+1} a_{i} & =a_{i+1} a_{i} a_{i+1}=0 & & \text { for all } 0 \leq i \leq N-1,
\end{aligned}
$$

where all indices are taken modulo $N$, so in particular $a_{N-1} a_{0} a_{N-1}=a_{0} a_{N-1} a_{0}=0$. The finite nilTemperley-Lieb algebra $\mathrm{nTL}_{N}$, as defined in [19], is the subalgebra of $\mathrm{nTL}_{N}$ generated by $a_{1}, \ldots, a_{N-1}$ (or in fact, by any $N-1$ of the generators $a_{i}$ ). We adopt the convention that $\mathrm{nTL}_{1}=\mathbb{k} 1$. We fix the following notation for monomials in $\mathrm{nTL}_{N}$ and $\mathrm{nTL}_{N}$ : For an ordered index sequence $\underline{j}=\left(j_{1}, \ldots, j_{m}\right)$ with $0 \leq j_{1}, \ldots, j_{m} \leq N-1$, we define the ordered monomial $a(j)=a_{j_{1}} \ldots a_{j_{m}}$. Unless otherwise specified, we use the letters $i, j$ for indices from $\mathbb{Z} / N \mathbb{Z}$; in particular, we often identify the indices 0 and $N$.

Throughout we will assume $N \geq 3$.

\section{Gradings}

One of the ingredients needed in Sect. 5 to study the center of $\widehat{n T L}_{N}$ is a grading on the algebra.

Gradings faciliate the computation of the center of an algebra, as the following standard result reduces the work to determining homogeneous central elements.

Lemma 3.1 If $A=\bigoplus_{g \in G} A_{g}$ is an algebra graded by some abelian group $G$, then the center of $A$ is homogeneous, i.e. it inherits the grading.

Proof Let $a=\sum_{g \in G} a_{g}$ be a central element of the graded algebra $A=\bigoplus_{g \in G} A_{g}$. We have for $b_{h} \in A_{h}$ that

$$
\sum_{g \in G} a_{g} b_{h}=a b_{h}=b_{h} a=\sum_{g \in G} b_{h} a_{g} .
$$


Since this equality must hold in every graded component, we get $a_{g} b_{h}=b_{h} a_{g}$ for all homogeneous elements $b_{h}$. Now take any element $b=\sum_{h \in G} b_{h}$ in $A$, then

$$
a_{g} b=\sum_{h \in G} a_{g} b_{h}=\sum_{h \in G} b_{h} a_{g}=b a_{g}
$$

hence $a_{g}$ is central.

Since the defining relations are homogeneous, both $\mathrm{nTL}_{N}$ and $n \mathrm{TL}_{N}$ have a $\mathbb{Z}$-grading by the length of a monomial, i.e. all generators $a_{i}$ have $\mathbb{Z}$-degree 1 . This can be refined to a $\mathbb{Z}^{N}$-grading by assigning to the generator $a_{i}$ the degree $\zeta_{i}$, the $i$ th standard basis vector in $\mathbb{Z}^{N}$. In either grading, we say that the degree 0 part of an element in $\widehat{n T L}_{N}$ or $n \mathrm{~nL}_{N}$ is its constant term.

The $\mathbb{Z}^{N}$-grading is finer than the $\mathbb{Z}$-grading in the sense that any $\mathbb{Z}$-graded component of degree different from 0 decomposes into a sum of $\mathbb{Z}^{N}$-graded components of strictly smaller dimension.

Remark 3.2 Why do we exclude the case of $N \leq 2$ from our considerations? For $N=1,2$, there are isomorphisms $\widehat{n T L}_{N} \cong \mathrm{nTL}_{N+1}$, and in these cases the center is uninteresting. The algebra $\widehat{n T L}_{1}$ is 2-dimensional and commutative; while $n \widehat{T L}_{2}$ has dimension 5 , and its center can be computed by hand making use of Lemma 3.1 and can be shown to be the $\mathbb{k}$-span of $1, a_{0} a_{1}, a_{1} a_{0}$.

Remark 3.3 The affine (or finite) Temperley-Lieb algebra, which has relations $a_{i} a_{j}=a_{j} a_{i}$ for $i-j \neq \pm 1(\bmod N), a_{i} a_{i \pm 1} a_{i}=a_{i}$, and $a_{i}^{2}=\delta a_{i}$ for some $\delta \in \mathbb{k}$, is a filtered algebra with respect to the length filtration. For this algebra, the $\ell$ th filtration space is generated by

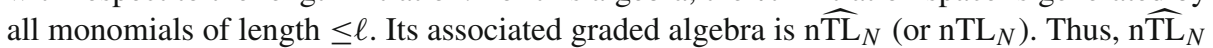
is infinite dimensional when $N \geq 3$, while $\mathrm{nTL}_{N}$ has dimension equal to the $N$ th Catalan number $\frac{1}{N+1}\left(\begin{array}{c}2 N \\ N\end{array}\right)$.

\section{A faithful representation}

The second ingredient we use to determine the center is a faithful representation of $\widehat{n T L}_{N}$. Here we recall the definition of the representation from [12] and describe its graphical realization, which is very convenient to work with.

Fix a basis $v_{1}, \ldots, v_{N}$ of $\mathbb{k}^{N}$. Consider the vector space $\mathrm{V}=\bigoplus_{k=0}^{N}\left(\mathbb{k}[q] \otimes \bigwedge^{k} \mathbb{k}^{N}\right)$. It has a standard $\mathbb{k}[q]$-basis consisting of wedges

$v(\mathbf{I}):=v_{i_{1}} \wedge \cdots \wedge v_{i_{k}}$ for all (strictly) increasing sequences $\mathbf{I}=\left\{1 \leq i_{1}<\cdots<i_{k} \leq N\right\}$

for all $0 \leq k \leq N$, where the basis element of $\mathbb{k}=\bigwedge^{0} \mathbb{k}^{N}$ is denoted $v(\emptyset)$. Throughout the rest of the paper, all tensor products are taken over $\mathbb{k}$, and we omit the tensor symbol in $\mathbb{k}[q]$-linear combinations of wedges.

Remark 4.1 The indices of the vectors $v_{j}$ should be interpreted modulo $N$. We make no distinction between $v_{0}$ and $v_{N}$ and often use the two interchangeably.

It is helpful to visualize the basis elements $v(\mathbf{I})$ as particle configurations having $0 \leq k \leq$ $N$ particles arranged on a circle with $N$ positions, where there is at most one particle at each site, as pictured below for $N=8$ and $v(1,5,6)=v_{1} \wedge v_{5} \wedge v_{6}$ (Fig. 2). The vector $v(\emptyset)$ 
Fig. 2 The element $v_{1} \wedge v_{5} \wedge v_{6}$ in the graphical realization

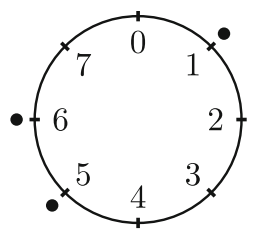

corresponds to the configuration with no particles. Then $\mathrm{V}$ is the $\mathbb{k}[q]$-span of such circular particle configurations.

There is an action of the affine nilTemperley-Lieb algebra $\widehat{n T L}_{N}$ defined on the basis vectors $v(\mathbf{I})$ of $\mathrm{V}$ as follows:

Definition 4.2 For $1 \leq j \leq N-1$,

$$
a_{j} v(\mathbf{I})= \begin{cases}v_{i_{1}} \wedge \cdots \wedge v_{i_{\ell-1}} \wedge v_{j+1} \wedge v_{i_{\ell+1}} \wedge \cdots \wedge v_{i_{k}}, & \text { if } i_{\ell}=j \text { for some } \ell, \\ 0, & \text { otherwise }\end{cases}
$$

For the action of $a_{0}$, note that $v_{N}$ appears in the basis element $v(\mathbf{I})$ if and only if it occurs in the last position, i.e. $v_{i_{k}}=v_{N}$, and define

$$
a_{0} v(\mathbf{I})= \begin{cases}(-1)^{k-1} q \cdot v_{1} \wedge v_{i_{1}} \wedge \cdots \wedge v_{i_{k-1}}, & \text { if } i_{k}=N, \\ 0, & \text { otherwise. }\end{cases}
$$

The sign appears in $a_{0} v(\mathbf{I})$ because of the equality

$$
q \cdot v_{i_{1}} \wedge \cdots \wedge v_{i_{k-1}} \wedge v_{1}=(-1)^{k-1} q \cdot v_{1} \wedge v_{i_{1}} \wedge \cdots \wedge v_{i_{k-1}} .
$$

Remark 4.3 It follows that $a_{j} v(\mathbf{I})=0$ if the sequence $\mathbf{I}$ contains $j+1$ or if it does not contain $j$. In other words, $a_{j}$ acts by replacing $v_{j}$ by $v_{j+1}$. If this creates a wedge expression with two factors equal to $v_{j+1}$, the result is zero. Thus, for any monomial $a(\underline{j})$ there is a unique increasing sequence $\mathbf{J}=\left\{1 \leq j_{1}<\cdots<j_{k} \leq N\right\}$ with $k$ minimal on which the monomial acts nontrivially.

In the graphical description, $a_{j}$ moves a particle clockwise from position $j$ to position $j+1$, and one records 'passing position 0 ' by multiplying by $\pm q$ as illustrated by the particle configurations in Fig. 3.

It is easy to verify that the defining relations for $\widehat{n T L}_{N}$ hold for this action, assuming that $N \geq 3$. Hence we obtain

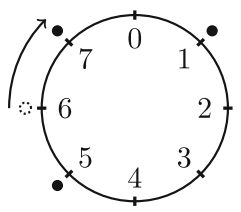

(a)

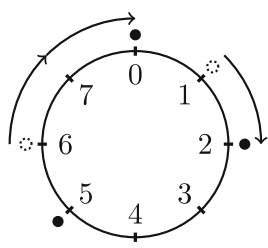

(b)

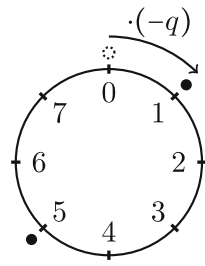

(c)

Fig. 3 Examples for the action of $\widehat{n}_{\mathrm{TL}_{N}}$ on a particle configuration. a $a_{6}\left(v_{1} \wedge v_{5} \wedge v_{6}\right)=v_{1} \wedge v_{5} \wedge v_{7}$, b $a_{7} a_{1} a_{6}\left(v_{1} \wedge v_{5} \wedge v_{6}\right)=v_{2} \wedge v_{5} \wedge v_{0}, \mathbf{c} a_{0}\left(v_{5} \wedge v_{0}\right)=-q \cdot v_{1} \wedge v_{5}$ 
Lemma 4.4 (a) Definition 4.2 gives a representation of $n \widehat{T L}_{N}$ on $\mathrm{V}$.

(b) The number of wedges (i.e., the number of particles) remains constant under the action of the generators $a_{i}$, so that $V=\bigoplus_{k=0}^{N}\left(\mathbb{k}[q] \otimes \bigwedge^{k} \mathbb{k}^{N}\right)$ is a direct sum decomposition of $V$ as an $n \widehat{T L}_{N}$-module.

The following crucial statement is taken from [2, Prop. 2.4.1] and [12, Prop. 9.1.(2)]. We will give a detailed proof adapted to our notation in Sect. 8.

Theorem 4.5 The action from Definition 4.2 gives a faithful representation of $n \widehat{T L}_{N}$ on $V$ when $N \geq 3$.

From now on, we will identify elements of $\widehat{n T L}_{N}$ with their action on the particle configurations of the graphical representation.

Remark 4.6 The spaces $\mathbb{k}[q] \otimes \bigwedge^{0} \mathbb{k}^{N}$ and $\mathbb{k}[q] \otimes \bigwedge^{N} \mathbb{k}^{N}$ are trivial summands in $\mathrm{V}$ on which every generator $a_{i}$ acts as 0 , and so they may be ignored when proving Theorem 4.5.

For a standard basis element $v(\mathbf{I})$ of $1 \leq k \leq N-1$ wedges corresponding to an increasing sequence $\mathbf{I}=\left\{1 \leq i_{1}<\cdots<i_{k} \leq N\right\}$, the next lemma defines a certain monomial $a(\hat{\mathbf{I}})$ that projects $v(\mathbf{I})$ onto $(-1)^{k-1} q v(\mathbf{I})$ and sends $v\left(\mathbf{I}^{\prime}\right)$ to zero for $\mathbf{I}^{\prime} \neq \mathbf{I}$. Before stating the result, we give an example to demonstrate in the graphical description how this projector will be defined.

Example 4.7 Let $N=8$, and consider the particle configuration $v(\mathbf{I})=v_{1} \wedge v_{5} \wedge v_{6}$. With $a(\widehat{156})=\left(a_{0} a_{7}\right) \cdot\left(a_{4} a_{3} a_{2}\right) \cdot\left(a_{1} a_{5} a_{6}\right)$ we obtain $a(\widehat{156}) \cdot v_{1} \wedge v_{5} \wedge v_{6}=(-1)^{2} q \cdot v_{1} \wedge v_{5} \wedge v_{6}$, which looks as follows in the graphical description (Fig. 4).

The factor $a_{1} a_{5} a_{6}$ moves every particle one step forward clockwise. It is critical that we start by moving the particle at position 6 before moving the particle at position 5 , as otherwise the result would be zero. But since there is a 'gap' at position 7, we can move the particle from site 6 to 7, and afterwards the particle from site 5 to 6 , without obtaining zero. The assumption that $k<N$ ensures such a gap always exists.

After applying $a_{1} a_{5} a_{6}$, the particles are at positions 2,6 , and 7 . The particle previously at position 5 is now at position 6 , which is where we want a particle to be. The particle currently at position 2 can be moved to position 5 by applying the product $a_{4} a_{3} a_{2}$. The particle now at position 7 can be moved by $a_{0} a_{7}$ to position 1 . Hence, the result of applying $\left(a_{0} a_{7}\right) \cdot\left(a_{4} a_{3} a_{2}\right) \cdot\left(a_{1} a_{5} a_{6}\right)$ is the same particle configuration as the original one. However, the answer must be multiplied by $\pm q$, since applying $a_{0} a_{7}$ involves crossing the zero position once. To determine the sign, note from Definition 4.2 that $\left(a_{0} a_{7}\right) \cdot\left(a_{4} a_{3} a_{2}\right) \cdot\left(a_{1} a_{5} a_{6}\right)\left(v_{1} \wedge\right.$ $\left.v_{5} \wedge v_{6}\right)=q \cdot v_{5} \wedge v_{6} \wedge v_{1}=(-1)^{2} q \cdot v_{1} \wedge v_{5} \wedge v_{6}$, so the sign is + .

Now we describe the general procedure:

Fig. 4 The action of $a(\widehat{156})$ on the particle configuration $v_{1} \wedge v_{5} \wedge v_{6}$

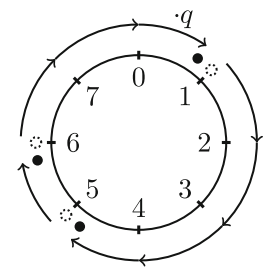


Lemma 4.8 Assume $v(\mathbf{I})$ is a particle configuration, where $\mathbf{I}=\left\{1 \leq i_{1}<\cdots<i_{k} \leq N\right\}$ is an increasing sequence and $1 \leq k \leq N-1$. Then there exists an index $\ell$ such that $i_{\ell}+1<i_{\ell+1}$ (or $\left.i_{k}+1<i_{1}\right)$, i.e. the sequence has a 'gap' between $i_{\ell}$ and $i_{\ell+1}$. Split the sequence $\mathbf{I}$ into the two parts $\left\{i_{1}<\cdots<i_{\ell}\right\}$ and $\left\{i_{\ell+1}<\cdots<i_{k}\right\}$. Set

$$
\begin{gathered}
a(\hat{\mathbf{I}}):=\left(a_{i_{1}-1} a_{i_{1}-2} \ldots a_{i_{k}+2} a_{i_{k}+1}\right) \cdot \prod_{s=1}^{k-1}\left(a_{i_{s+1}-1} a_{i_{s+1}-2} \ldots a_{i_{s}+2} a_{i_{s}+1}\right) \\
\cdot\left(a_{i_{\ell+1}} a_{i_{\ell+2}} \ldots a_{i_{k-1}} a_{i_{k}}\right) \cdot\left(a_{i_{1}} a_{i_{2}} \ldots a_{i_{\ell-1}} a_{i_{\ell}}\right)
\end{gathered}
$$

where the indices are modulo $N$ in the factor $\left(a_{i_{1}-1} a_{i_{1}-2} \ldots a_{i_{k}+2} a_{i_{k}+1}\right)$. Then

$$
a(\hat{\mathbf{I}}) v\left(\mathbf{I}^{\prime}\right)= \begin{cases}(-1)^{k-1} q \cdot v(\mathbf{I}) & \text { if } \mathbf{I}^{\prime}=\mathbf{I}, \\ 0 & \text { for all } \mathbf{I}^{\prime} \neq \mathbf{I} \text { (of any length), }\end{cases}
$$

and $a(\hat{\mathbf{I}})$ has $\mathbb{Z}^{N}$-degree $(1,1, \ldots, 1)$.

Proof The assertions can be seen using the graphical realization of $\mathrm{V}$. The terms in the second line of equation (*) move a particle at site $i_{j} \in \mathbf{I}$ one step forward to $i_{j}+1$ for each $j$, while the terms in the first line send the particle from $i_{j}+1$ to the original position of $i_{j+1}$.

Consider first $a(\hat{\mathbf{I}}) v(\mathbf{I})$. By applying $\left(a_{i_{\ell+1}} a_{i_{\ell+2}} \ldots a_{i_{k-1}} a_{i_{k}}\right) \cdot\left(a_{i_{1}} a_{i_{2}} \ldots a_{i_{\ell-1}} a_{i_{\ell}}\right)$, every particle is first moved clockwise by one position. By our choice of the index $i_{\ell}$, we avoid mapping the whole particle configuration to zero. After that step, every particle is moved by one of the factors $\left(a_{i_{s+1}-1} a_{i_{s+1}-2} \ldots a_{i_{s}+2} a_{i_{s}+1}\right)$ to the original position of its successor in the sequence $\mathbf{I}$, so the particle configuration remains the same. One of the particles has passed the zero position, so we have to multiply by $\pm q$. Definition 4.2 tells us the appropriate sign is $(-1)^{k-1}$.

Now consider $a(\hat{\mathbf{I}}) v\left(\mathbf{I}^{\prime}\right)$ for $\mathbf{I}^{\prime} \neq \mathbf{I}$. The monomial $\left(a_{i_{\ell+1}} a_{i_{\ell+2}} \ldots a_{i_{k-1}} a_{i_{k}}\right) \cdot\left(a_{i_{1}} a_{i_{2}} \ldots\right.$ $a_{i_{\ell-1}} a_{i_{\ell}}$ ) expects a particle at each of the sites $i_{1}, \ldots, i_{k}$, so if any of these positions is empty in $v\left(\mathbf{I}^{\prime}\right)$, the result of applying $a(\hat{\mathbf{I}})$ is zero. If the positions $i_{1}, \ldots, i_{k}$ are already filled, and there is an additional particle somewhere, multiplication by $\left(a_{i_{\ell+1}-1} a_{i_{\ell+1}}-2 \ldots a_{i_{\ell}+2} a_{i_{\ell}+1}\right)$ will cause two particles to be at the same position, hence the result is again zero.

Since every $a_{j}$ appears in $a(\hat{\mathbf{I}})$ exactly once, the monomial $a(\hat{\mathbf{I}})$ has $\mathbb{Z}^{N}$-degree $(1,1, \ldots, 1)$.

Example 4.9 In the previous example, $N=8, \mathbf{I}=(1,5,6)$, and we may assume the two subsequences are $(1)$ and $(5,6)$. Then the terms in the second line of $(*)$ are $\left(a_{5} a_{6}\right) \cdot\left(a_{1}\right)=$ $a_{1} a_{5} a_{6}$. The term corresponding to $j=1$ in the product on the first line of (*) is $a_{4} a_{3} a_{2}$, and the expression corresponding to $j=2$ is empty, hence taken to be 1 . The first factor on the first line is $a_{0} a_{7}$. Thus, for $\mathbf{I}=(1,5,6), a(\hat{\mathbf{I}})=\left(a_{0} a_{7}\right) \cdot\left(a_{4} a_{3} a_{2}\right) \cdot\left(a_{1} a_{5} a_{6}\right)$, as in Example 4.7. If the gap between 6 and 0 is used instead, the right-hand factor of the second line is $a_{1} a_{5} a_{6}$ and the left-hand factor is 1 . The factors in the first line remain the same, and so one obtains the same expression for $a(\hat{\mathbf{I}})$.

Remark 4.10 Because $\mathrm{V}$ is a faithful module, $a(\hat{\mathbf{I}})$ is, as an element in $\mathrm{nTL}_{N}$ (i.e. up to reordering according to the defining relations), uniquely determined by the increasing sequence I. One can read off I from $a(\hat{\mathbf{I}})$ as follows: In the defining equation $(*)$ of $a(\hat{\mathbf{I}})$, the factors in the first line are pairwise commuting. The underlying subsequence $\left(i_{s+1}-1, i_{s+1}-2, \ldots, i_{s}+2, i_{s}+1\right)$ corresponding to the factor $a_{i_{s+1}-1} a_{i_{s+1}}-2 \ldots a_{i_{s}+2} a_{i_{s}+1}$ of $a(\hat{\mathbf{I}})$ is a decreasing sequence. After all such decreasing sequences are removed from $a(\hat{\mathbf{I}})$, what remains is a product of generators $a_{j}$ with an increasing subsequence of indices or a 
product of two such subsequences corresponding to the factors in the second line. This is $\mathbf{I}$. Given any monomial $a(\underline{r})$ of $\mathbb{Z}^{N}$-degree $(1, \ldots, 1)$, one can rewrite it using the relations in $\mathrm{nTL}_{N}$ so that it is of the form $a(\hat{\mathbf{I}})$ for some increasing sequence $\mathbf{I}$. Then $v(\mathbf{I})$ is the unique standard basis element upon which $a(\underline{r})=a(\hat{\mathbf{I}})$ acts by multiplication by $\pm q$.

\section{Description of the center}

In this section, we give an explicit description of the center $\mathrm{C}_{N}$ of $\mathrm{n} \widehat{\mathrm{TL}}_{N}$. We start with the following initial characterisation of the central elements:

Lemma 5.1 Any central element $c$ in $n \widehat{T L}_{N}$ with constant term 0 is a linear combination of monomials $a(j)=a_{j_{1}} \cdots a_{j_{m}}$ where every generator $a_{i}, 0 \leq i \leq N-1$, appears at least once. In particular, a homogeneous nonconstant central element c has $\mathbb{Z}$-degree at least $N$.

Proof Assume $c=\sum_{\underline{j}} c_{\underline{j}} a(\underline{j})$, where $c_{\underline{j}} \in \mathbb{k}$ for all $\underline{j}$. By Lemma 3.1, we can assume $c$ is a homogeneous central element with respect to the $\mathbb{Z}^{N}$-grading. By our assumption, $c \notin \mathbb{k}$. For all $i$, we need to show that $a_{i}$ occurs in each monomial $a(j)$ appearing in $c$. Without loss of generality, we show this for $i=0$. If some summand is missing $a_{0}$, then no summand contains $a_{0}$ because $c$ is homogeneous. Hence $a_{0} a(j) \neq 0$ and $a(\underline{j}) a_{0} \neq 0$ for all $\underline{j}$ with $c_{j} \neq 0$, and since $a_{0} c=c a_{0}$, none of the $a(j)$ can contain the factor $a_{1}$ either, as otherwise the factor $a_{0}$ cannot pass through $c$ from left to right (so also $a_{N-1}$ cannot be contained in the $a(j)$ ). Proceeding inductively, we see that all $a(j)$ must be a constant, contrary to our assumption.

The next proposition states that on the standard wedge basis vector $v(\mathbf{I})$ of $\mathrm{V}$, any central element acts via multiplication by a polynomial $p_{k} \in \mathbb{K}[q]$ that only depends on the length $k=|\mathbf{I}|$ of the increasing sequence $\mathbf{I}=\left\{1 \leq i_{1}<\cdots<i_{k} \leq N\right\}$. In other words, the decomposition of $\mathrm{V}$ into the summands $\mathbb{k}[q] \otimes \bigwedge^{k} \mathbb{k}^{N}$ is a decomposition with respect to different central characters (apart from the two trivial summands for $k \in\{0, N\}$ ).

Proposition 5.2 For any central element $c \in n \widehat{T L}_{N}$ and all increasing sequences $\mathbf{I}$ with fixed length $k$, there is some element $p_{k} \in \mathbb{k}[q]$ such that $c v(\mathbf{I})=p_{k} v(\mathbf{I})$.

Proof We may assume $c$ is a nonconstant $\mathbb{Z}^{N}$-homogeneous central element of $n \widehat{\mathrm{TL}}_{N}$. For $k \in\{0, N\}$, the action of a generator $a_{i}$ on a monomial of length $k$ is 0 , so $p_{k}=0$ for such values of $k$. Now consider $1 \leq k \leq N-1$, and suppose that $\mathbf{I}=\left\{1 \leq i_{1}<\cdots<i_{k} \leq N\right\}$ is an increasing sequence of length $k$. According to Lemma 4.4(b), the number of wedges in a vector remains constant under the action of the $a_{i}$. Hence $c v(\mathbf{I})=\sum_{\left|\mathbf{I}^{\prime}\right|=k} c_{\mathbf{I}^{\prime}} v\left(\mathbf{I}^{\prime}\right)$ for some polynomials $c_{\mathbf{I}^{\prime}} \in \mathbb{k}[q]$. We want to prove that $c_{\mathbf{I}^{\prime}}=0$ for all $\mathbf{I}^{\prime} \neq \mathbf{I}$.

We have shown in Lemma 4.8 that to each increasing sequence $\mathbf{J} \subset\{1, \ldots, N\}$ there corresponds a monomial $a(\hat{\mathbf{J}}) \in \mathrm{nTL}_{N}$ that allows us to select a single basis vector:

$$
a(\hat{\mathbf{J}}) v(\mathbf{I})= \begin{cases}(-1)^{k-1} q v(\mathbf{J}) & \text { if } \mathbf{I}=\mathbf{J}, \\ 0 & \text { otherwise. }\end{cases}
$$

Thus, for $\mathbf{J} \neq \mathbf{I}$, we see that

$$
0=c(a(\hat{\mathbf{J}}) v(\mathbf{I}))=a(\hat{\mathbf{J}})(c v(\mathbf{I}))=a(\hat{\mathbf{J}})\left(\sum_{\left|\mathbf{I}^{\prime}\right|=k} c_{\mathbf{I}^{\prime}} v\left(\mathbf{I}^{\prime}\right)\right)=c_{\mathbf{J}}(-1)^{k-1} q v(\mathbf{J}),
$$


implying $c_{\mathbf{J}}=0$ for $\mathbf{J} \neq \mathbf{I}$. Hence, we may assume for each increasing sequence $\mathbf{I}$ that $c v(\mathbf{I})=p_{\mathbf{I}} v(\mathbf{I})$ for some polynomial $p_{\mathbf{I}} \in \mathbb{k}[q]$.

Now it is left to show that $p_{\mathbf{I}}=p_{\mathbf{I}^{\prime}}$ for all $\mathbf{I}^{\prime}$ with $\left|\mathbf{I}^{\prime}\right|=|\mathbf{I}|=k$. It is enough to verify this for $\mathbf{I}, \mathbf{I}^{\prime}$ that differ in exactly one entry, i.e. $i_{s}=i, i_{s}^{\prime}=i+1$, and $i_{\ell}=i_{\ell}^{\prime}$ for all $\ell \neq s$, for some $1 \leq s \leq k$ and $i \in \mathbb{Z} / N \mathbb{Z}$. If $1 \leq i \leq N-1$, we have

$$
p_{\mathbf{I}^{\prime}} v\left(\mathbf{I}^{\prime}\right)=c v\left(\mathbf{I}^{\prime}\right)=c\left(a_{i} v(\mathbf{I})\right)=a_{i}(c v(\mathbf{I}))=a_{i}\left(p_{\mathbf{I}} v(\mathbf{I})\right)=p_{\mathbf{I}} v\left(\mathbf{I}^{\prime}\right),
$$

and if $i=0$, we get

$$
\begin{aligned}
(-1)^{k-1} q p_{\mathbf{I}^{\prime}} v\left(\mathbf{I}^{\prime}\right) & =(-1)^{k-1} q c v\left(\mathbf{I}^{\prime}\right)=c\left(a_{0} v(\mathbf{I})\right)=a_{0}(c v(\mathbf{I}))=a_{0}\left(p_{\mathbf{I}} v(\mathbf{I})\right) \\
& =(-1)^{k-1} q p_{\mathbf{I}} v\left(\mathbf{I}^{\prime}\right) .
\end{aligned}
$$

Hence, $p_{\mathbf{I}^{\prime}}=p_{\mathbf{I}}$, and this common polynomial is the desired polynomial $p_{k}$.

Corollary 5.3 Any central element in $n \widehat{T L}_{N}$ with constant term 0 acts on a standard basis vector $v(\mathbf{I}) \in V$ as multiplication by an element of $q \mathbb{k}[q]$.

Proof According to Lemma 5.1, each summand of such a central element must contain the factor $a_{0}$, and $a_{0}$ acts on a wedge product by 0 or multiplication by $\pm q$.

Now we are ready to introduce nontrivial central elements in $\widehat{n T L}_{N}$. For each $1 \leq k \leq$ $N-1$, set

$$
\mathbf{t}_{k}:=(-1)^{k-1} \sum_{|\mathbf{I}|=k} a(\hat{\mathbf{I}}),
$$

where the monomials $a(\hat{\mathbf{I}})$ correspond to increasing sequences $\mathbf{I}=\left\{1 \leq i_{1}<\cdots<i_{k} \leq N\right\}$ of length $k$ as defined in Lemma 4.8 .

Example 5.4 In $\widehat{n T L}_{3}$ :

$$
\begin{aligned}
& \mathbf{t}_{1}=a_{2} a_{1} a_{0}+a_{0} a_{2} a_{1}+a_{1} a_{0} a_{2}, \\
& \mathbf{t}_{2}=-a_{0} a_{1} a_{2}-a_{1} a_{2} a_{0}-a_{2} a_{0} a_{1} .
\end{aligned}
$$

In $\widehat{\mathrm{TL}}_{4}$ :

$$
\begin{aligned}
& \mathbf{t}_{1}=a_{3} a_{2} a_{1} a_{0}+a_{0} a_{3} a_{2} a_{1}+a_{1} a_{0} a_{3} a_{2}+a_{2} a_{1} a_{0} a_{3}, \\
& \mathbf{t}_{2}=-a_{0} a_{3} a_{1} a_{2}-a_{0} a_{2} a_{1} a_{3}-a_{3} a_{2} a_{0} a_{1}-a_{1} a_{0} a_{2} a_{3}-a_{1} a_{3} a_{0} a_{2}-a_{2} a_{1} a_{3} a_{0} \\
& \mathbf{t}_{3}=a_{0} a_{1} a_{2} a_{3}+a_{1} a_{2} a_{3} a_{0}+a_{2} a_{3} a_{0} a_{1}+a_{3} a_{0} a_{1} a_{2} .
\end{aligned}
$$

In the graphical realization of $\mathrm{V}, \mathbf{t}_{k}$ acts by annihilating all particle configurations whose number of particles is different from $k$. For particle configurations having $k$ particles, every particle is moved clockwise to the original site of the next particle. Hence, the particle configuration itself remains fixed by the action of $\mathbf{t}_{k}$ (and it is multiplied with $(-1)^{2(k-1)} q=$ $q$, since a particle has been moved through position 0$)$. All the $\mathbf{t}_{k}$ have $\mathbb{Z}^{N}$-degree equal to $(1, \ldots, 1)$ and $\mathbb{Z}$-degree equal to $N$. Any monomial whose $\mathbb{Z}^{N}$-degree is $(1, \ldots, 1)$ occurs as a summand in some central element (after possibly reordering the factors), and the number of summands of $\mathbf{t}_{k}$ equals $\left(\begin{array}{l}N \\ k\end{array}\right)=\operatorname{dim}\left(\bigwedge^{k} \mathbb{k}^{N}\right)$; see Remark 4.10.

Theorem 5.5 1. The $t_{k}$ are central for all $1 \leq k \leq N-1$, and the center of $n \widehat{T L}_{N}$ is generated by 1 and the $t_{k}, 1 \leq k \leq N-1$. 
2. The subalgebra generated by $\boldsymbol{t}_{k}$ is isomorphic to the polynomial ring $\mathbb{k}[q]$ for all $1 \leq$ $k \leq N-1$. Moreover $\boldsymbol{t}_{k} \boldsymbol{t}_{\ell}=0$ for all $k \neq \ell$. Hence the center of $n \widehat{T L}_{N}$ is the subalgebra

$$
C_{N}=\mathbb{k} \oplus \boldsymbol{t}_{1} \mathbb{k}\left[\boldsymbol{t}_{1}\right] \oplus \cdots \oplus \boldsymbol{t}_{N-1} \mathbb{k}\left[\boldsymbol{t}_{N-1}\right] \cong \frac{\mathbb{k}\left[\boldsymbol{t}_{1}, \ldots, \boldsymbol{t}_{N-1}\right]}{\left(\boldsymbol{t}_{k} \boldsymbol{t}_{\ell} \mid k \neq \ell\right)} .
$$

Proof 1. The action of $\mathbf{t}_{k}$ on $\mathrm{V}$ is the projection onto the $\mathrm{nTL}_{N}$-submodule $\mathbb{k}[q] \otimes \bigwedge^{k} \mathbb{k}^{N}$ followed by multiplication by $q$. This commutes with the action of every other element of $n \widehat{T L}_{N}$. Since $\mathrm{V}$ is a faithful module, $\mathbf{t}_{k}$ commutes with any element of $\mathrm{nTL}_{N}$. As we have seen in Proposition 5.2, any central element $c$ without constant term acts on the summand $\mathbb{k}[q] \otimes \bigwedge^{k} \mathbb{k}^{N}$ via multiplication by some polynomial $p_{k}^{c} \in q \mathbb{k}[q]$. Once again using the faithfulness of $\mathrm{V}$, we get that $c=\sum_{k=1}^{N-1} p_{k}^{c}\left(\mathbf{t}_{k}\right)$.

2. Recall that $\mathbb{k}[q] \otimes \bigwedge^{k} \mathbb{k}^{N}$ is a free $\mathbb{k}[q]$-module of rank $\left(\begin{array}{c}N \\ k\end{array}\right)$. Since $\mathbf{t}_{k}$ acts by multiplication with $q$ on that module, the subalgebra of $\widehat{n T L}_{N}$ generated by $\mathbf{t}_{k}$ must be isomorphic to the polynomial ring $\mathbb{k}[q]$. Since $a(\hat{\mathbf{J}}) a(\hat{\mathbf{I}})=0$ for all $\mathbf{J} \neq \mathbf{I}$, we get $\mathbf{t}_{k} \mathbf{t}_{\ell}=0$ for $k \neq \ell$, as they consist of pairwise distinct summands.

Theorem 5.5 enables us to describe the $\mathbb{k}$-algebra $\operatorname{End}_{\mathrm{n} \widehat{T L}_{N}}(\mathrm{~W})$ of $\mathrm{n} \widehat{T L}_{N}$-endomorphisms of the space of nontrivial particle configurations $\mathbf{W}:=\bigoplus_{k=1}^{N-1}\left(\mathbb{k}[q] \otimes \bigwedge^{k} \mathbb{k}^{N}\right) \subset \mathrm{V}$. We first observe that on $\mathrm{W}$ multiplication by $q$ is given by the action of a central element in $\mathrm{C}_{N}$, therefore it is justified to speak about $\mathbb{k}[q]$-linearity of a $\widehat{n T L}_{N}$-endomorphism of $\mathrm{W}$.

Lemma 5.6 $\operatorname{End}_{n \widehat{T L}_{N}}(W) \subset \operatorname{End}_{\mathbb{k}[q]}(W)$, hence any $n \widehat{T L}_{N}$-module endomorphism $\varphi$ of $W$ is $\mathbb{k}[q]$-linear.

Proof Observe that $\sum_{k=1}^{N-1} \mathbf{t}_{k} \in \mathrm{nTL}_{N}$ acts by multiplication by $q$ on every element in $\mathrm{W}$. Therefore multiplication by $q$ commutes with the application of every $\varphi \in \operatorname{End}_{\mathrm{n} \widehat{T L}_{N}}(\mathrm{~W})$.

Proposition 5.7 The endomorphism algebra $\operatorname{End}_{n \widehat{T L}}(W)$ is isomorphic to a direct sum of $N-1$ polynomial algebras $\mathbb{k}\left[T_{1}\right] \oplus \cdots \oplus \mathbb{k}\left[T_{N-1}\right]$.

Proof The proof is very similar to that of Proposition 5.2. First we show that $\varphi(v(\mathbf{I}))$ is a $\mathbb{k}[q]$-linear multiple of $v(\mathbf{I})$ for any $\varphi \in \operatorname{End}_{\mathrm{nTL}_{N}}(\mathrm{~W})$ and any increasing sequence $\mathbf{I}$. This statement holds if and only if $\pm q \varphi(v(\mathbf{I})) \in \mathbb{k}[q] v(\mathbf{I})$. Indeed, by Lemmas 4.8 and 5.6 we get

$$
\pm q \varphi(v(\mathbf{I}))=\varphi( \pm q v(\mathbf{I}))=\varphi(a(\hat{\mathbf{I}}) v(\mathbf{I}))=a(\hat{\mathbf{I}}) \varphi(v(\mathbf{I})) \in \mathbb{k}[q] v(\mathbf{I}) .
$$

Therefore, we can write $\varphi(v(\mathbf{I}))=p_{\mathbf{I}} \cdot v(\mathbf{I})$ for some polynomial $p_{\mathbf{I}} \in \mathbb{k}[q]$. Note that this implies

$$
\operatorname{End}_{n} \widehat{\mathrm{TL}}_{N}\left(\bigoplus_{k=1}^{N-1}\left(\mathbb{k}[q] \otimes \bigwedge^{k} \mathbb{k}^{N}\right)\right)=\bigoplus_{k=1}^{N-1}\left(\operatorname{End}_{\mathrm{n} \widehat{T L}_{N}}\left(\mathbb{k}[q] \otimes \bigwedge^{k} \mathbb{k}^{N}\right)\right) .
$$

What remains is to show that these polynomials only depend on the number of particles in $\mathbf{I}$, in other words there exists $p_{k} \in \mathbb{k}[q]$ so that $p_{\mathbf{I}}=p_{k}$ for all $\mathbf{I}$ with $|\mathbf{I}|=k$. Again it suffices to show this for two sequences $\mathbf{I}, \mathbf{I}^{\prime}$ of length $k$ that differ in exactly one entry. So say $i_{s}=i, i_{s}^{\prime}=i+1$, and $i_{\ell}=i_{\ell}^{\prime}$ for all $\ell \neq s$, for some $1 \leq s \leq k$ and $i \in \mathbb{Z} / N \mathbb{Z}$. When $1 \leq i \leq N-1$,

$$
p_{\mathbf{I}^{\prime}} v\left(\mathbf{I}^{\prime}\right)=\varphi\left(v\left(\mathbf{I}^{\prime}\right)\right)=\varphi\left(a_{i} v(\mathbf{I})\right)=a_{i} \varphi(v(\mathbf{I}))=a_{i}\left(p_{\mathbf{I}} v(\mathbf{I})\right)=p_{\mathbf{I}} v\left(\mathbf{I}^{\prime}\right),
$$


and when $i=0$,

$$
\begin{aligned}
(-1)^{k-1} q p_{\mathbf{I}^{\prime}} v\left(\mathbf{I}^{\prime}\right) & =(-1)^{k-1} q \varphi\left(v\left(\mathbf{I}^{\prime}\right)\right)=\varphi\left(a_{0} v(\mathbf{I})\right)=a_{0} \varphi(v(\mathbf{I}))=a_{0}\left(p_{\mathbf{I}} v(\mathbf{I})\right) \\
& =(-1)^{k-1} q p_{\mathbf{I}} v\left(\mathbf{I}^{\prime}\right) .
\end{aligned}
$$

Hence we can write $\varphi=\sum_{k=1}^{N-1} p_{k} \pi_{k}$ where $\pi_{k}$ is the projection onto $\mathbb{k}[q] \otimes \bigwedge^{k} \mathbb{k}^{N}$, and we get that

$$
\operatorname{End}_{\mathrm{nTL}}\left(\mathbb{k}[q] \otimes \bigwedge^{k} \mathbb{k}^{N}\right)=\mathbb{k}\left[T_{k}\right],
$$

where $T_{k}$ denotes the multiplication action of the central element $\mathbf{t}_{k}$, which is indeed a $\widehat{n T L}_{N^{-}}$ module endomorphism of W. Thus, $\operatorname{End}_{\mathrm{nTL}_{N}}(\mathrm{~W})$ is isomorphic to a direct sum of polynomial algebras as claimed.

Remark 5.8 The arguments in the proof of Proposition 5.7 remain valid even if we specialize the indeterminate $q$ to some element in $\mathbb{k} \backslash\{0\}$. In this case, we obtain that the summands $\bigwedge^{k} \mathbb{k}^{N}$ are simple modules and $\operatorname{End}_{\mathrm{n} \widehat{T L}_{N}}\left(\bigoplus_{k=1}^{N-1} \bigwedge^{k} \mathbb{k}^{N}\right) \cong \mathbb{k}^{N-1}$. For $q=0$, the situation is more complicated: If $q$ is specialized to zero, the generator $a_{0}$ acts by zero on the module. The action of $\widehat{n T L}_{N}$ factorizes over $\mathrm{nTL}_{N}$, and the module $\bigwedge^{k} \mathbb{k}^{N}$ is no longer simple. Instead it has a one-dimensional head spanned by the particle configuration $v(1, \ldots, k)$, and any endomorphism is given by choosing an image of this top configuration. It is always possible to map it to itself and to the one-dimensional socle spanned by $v(N-k, \ldots, N)$, but in general there are more endomorphisms. For example, in $\bigwedge^{4} \mathbb{k}^{8}$, the image of $v(1,2,3,4)$ may be any linear combination of $v(1,2,3,4), v(2,3,4,8), v(3,4,7,8), v(4,6,7,8)$ and $v(5,6,7,8)$, so that $\operatorname{End}_{\mathrm{n} \widehat{T L}_{8}}\left(\bigwedge^{4} \mathbb{k}^{8}\right)$ is 5-dimensional.

\section{The affine nilTemperley-Lieb algebra is finitely generated over its center}

The affine nilTemperley-Lieb algebra is infinite dimensional when $N \geq 3$; however, the following finiteness result holds:

Theorem 6.1 The algebra $n \widehat{T L}_{N}$ is finitely generated over its center.

Proof Given an arbitrary monomial $a(j) \in \mathrm{nTL}_{N}$, we first factor it as $a\left(\underline{j}^{\prime}\right) \cdot a\left(\underline{j}^{(0)}\right)$ in the following way: Take the minimal particle configuration $\mathbf{J}=\left\{1 \leq j_{1}<\cdots<j_{k} \leq N\right\}$ on which the monomial $a(j)$ acts nontrivially; see Remark 4.3. The monomial $a(j)$ moves all of the particles by at least one step, because the particle configuration was assumed to be minimal. Using the faithfulness of the representation, we know that we may reorder the monomial $a(j)$ so that first each particle is moved one step clockwise, and afterwards the remaining particle moves are carried out. Hence, we may choose some factorization $a(j)=a\left(\underline{j}^{\prime}\right) \cdot a\left(\underline{j}^{(0)}\right)$, where $\underline{j}^{(0)}$ is a sequence obtained by permuting $j_{1}, \ldots, j_{k}$ so that the particle at position $j_{r}$ is moved one step clockwise by the action of $a_{j_{r}}$ for all $1 \leq r \leq k$. The remaining particle moves are carried out by $a\left(\underline{j^{\prime}}\right)$.

In Sect. 8, this decomposition is explicitly constructed (not using the faithful representation).

Next, we want to find an expression of the form

$$
a(\underline{j})=a_{\text {fin }} \cdot \mathbf{t}_{k}^{n} \cdot a\left(\underline{j}^{(0)}\right),
$$


where $a_{\mathrm{fin}}$ is a monomial of some subalgebra ${ }^{i} \mathrm{nTL}_{N}$ of $\mathrm{nTL}_{N}, \mathbf{t}_{k}^{n}$ is in the center of $\widehat{n T L}_{N}$, and $a\left(\underline{j}^{(0)}\right)$ is the above factor. Here

$$
{ }^{i} \mathrm{nTL}_{N}=\left\langle a_{0}, \ldots, a_{i-1}, a_{i+1}, \ldots, a_{N-1}\right\rangle
$$

denotes a copy of the finite nilTemperley-Lieb algebra $\mathrm{nTL}_{N}$ sitting in $\mathrm{n} \widehat{\mathrm{TL}}_{N}$. To accomplish this, we have to subdivide the action of $a(j)$ on the particle configuration $\mathbf{J}=\left\{j_{1}<\cdots<j_{k}\right\}$ one more time. There are two cases:

1. There is an index $i$ not appearing in $\underline{j^{\prime}}$. In this case, $a\left(\underline{j^{\prime}}\right)$ is an element of ${ }^{i} \mathrm{nTL}_{N}$ and we are done.

2. All indices appear at least $n \geq 1$ times in $\underline{j^{\prime}}$. Let us investigate the action of $a\left(j^{\prime}\right)$ on

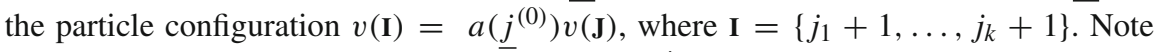
that $\mathbf{I}$ is the minimal particle configuration for $a\left(j^{\prime}\right)$. Each of the particles in $\mathbf{I}$ is moved by $a\left(j^{\prime}\right)$ to the position of the next particle in the sequence $\mathbf{I}$, because there is no index missing (a missing index is equivalent to a particle being stopped before reaching the position of its successor), before possibly continuing to move along the circle. Again invoking the faithfulness of the representation, we can rewrite $a\left(\underline{j}^{\prime}\right)=a\left(\underline{j^{\prime \prime}}\right) \cdot a(\hat{\mathbf{I}})^{n}$, with the monomial $a(\hat{\mathbf{I}})$ from Lemma 4.8. For maximal $n$, the remaining factor $a\left(j^{\prime \prime}\right)$ is an element of ${ }^{i} \mathrm{nTL}_{N}$ for some $i$. Observe that $a(\hat{\mathbf{I}})^{n} a\left(\underline{j}^{(0)}\right)=\mathbf{t}_{k}^{n} a\left(\underline{j}^{(0)}\right)$, which follows immediately from the definition of $\mathbf{t}_{k}$ and Lemma 4.8 .

Therefore, we have shown that

$$
a(\underline{j})=a\left(\underline{j}^{\prime}\right) \cdot a\left(\underline{j}^{(0)}\right)=a_{\mathrm{fin}} \cdot a(\hat{\mathbf{I}})^{n} \cdot a\left(\underline{j}^{(0)}\right)=a_{\mathrm{fin}} \cdot \mathbf{t}_{k}^{n} \cdot a\left(\underline{j}^{(0)}\right),
$$

where $n=0$ in the first case. Since there are only finitely many monomials in ${ }^{0} \mathrm{nTL}_{N},{ }^{1} \mathrm{nTL}_{N}, \ldots,{ }^{N-1} \mathrm{nTL}_{N}$ and only finitely many monomials $a\left(j^{(0)}\right)$ such that every index $0,1, \ldots, N-1$ occurs at most once in the sequence $a\left(\underline{j}^{(0)}\right)$, the affine nilTemperleyLieb algebra is indeed finitely generated over its center.

Remark 6.2 The affine nilTemperley-Lieb algebra is not free over its center (see [18]).

\section{Embeddings of affine nilTemperley-Lieb algebras}

In the proof of Theorem 6.1, we have used the $N$ obvious embeddings of $\mathrm{nTL}_{N}$ into $\widehat{\mathrm{TL}}_{N}$ coming from the $N$ different embeddings of the Coxeter graph $\mathrm{A}_{N-1}$ into $\tilde{\mathrm{A}}_{N-1}$. Next we construct $N$ embeddings of $\mathrm{nTL}_{N}$ into $\widehat{n T L}_{N+1}$. They correspond to the subdivision of an edge of $\tilde{\mathrm{A}}_{N-1}$ by inserting a vertex on the edge to obtain $\tilde{\mathrm{A}}_{N}$.

Theorem 7.1 Let $N \geq 3$. For any number $0 \leq m \leq N-1$, there is a unital embedding of algebras $\varepsilon_{m}: n \widehat{T L}_{N} \rightarrow n \widehat{T L}_{N+1}$ given by

$$
a_{i} \mapsto \begin{cases}a_{i} & \text { for } 0 \leq i \leq m-1, \\ a_{m+1} a_{m} & \text { for } i=m, \\ a_{i+1} & \text { for } m+1 \leq i \leq N-1 .\end{cases}
$$

Lemma 7.2 For $N \geq 3$, the map $\varepsilon_{m}$ from $n \widehat{T L}_{N}$ to $n \widehat{T L}_{N+1}$ given by (3) is an algebra homomorphism. 
Fig. $5 \varepsilon_{5}\left(\mathrm{n} \widehat{\mathrm{TL}}_{7}\right) \subset \mathrm{n} \widehat{\mathrm{TL}}_{8}$ : The action of $\varepsilon_{5}\left(a_{0} a_{6} a_{5} a_{4}\right)=$ $\tilde{a}_{0} \tilde{a}_{7} \tilde{a}_{6} \tilde{a}_{5} \tilde{a}_{4}$ on the particle configuration $v(4)$

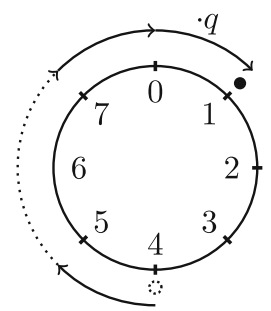

Proof Due to the circular nature of the relations, it suffices to check this for $\varepsilon_{0}$. This amounts to showing the following, since all the other relations are readily apparent. To avoid confusion, we indicate generators of $\widehat{n T L}_{N+1}$ in these calculations by $\tilde{a}_{i}$ :

$\left(\tilde{a}_{1} \tilde{a}_{0}\right)\left(\tilde{a}_{1} \tilde{a}_{0}\right)=\tilde{a}_{1}\left(\tilde{a}_{0} \tilde{a}_{1} \tilde{a}_{0}\right)=0, \quad \tilde{a}_{2}\left(\tilde{a}_{1} \tilde{a}_{0}\right) \tilde{a}_{2}=\left(\tilde{a}_{2} \tilde{a}_{1} \tilde{a}_{2}\right) \tilde{a}_{0}=0, \quad \tilde{a}_{N}\left(\tilde{a}_{1} \tilde{a}_{0}\right) \tilde{a}_{N}=\tilde{a}_{1}\left(\tilde{a}_{N} \tilde{a}_{0} \tilde{a}_{N}\right)=0$, $\left(\tilde{a}_{1} \tilde{a}_{0}\right) \tilde{a}_{2}\left(\tilde{a}_{1} \tilde{a}_{0}\right)=\left(\tilde{a}_{1} \tilde{a}_{2}\right)\left(\tilde{a}_{0} \tilde{a}_{1} \tilde{a}_{0}\right)=0, \quad\left(\tilde{a}_{1} \tilde{a}_{0}\right) \tilde{a}_{N}\left(\tilde{a}_{1} \tilde{a}_{0}\right)=\left(\tilde{a}_{1} \tilde{a}_{0} \tilde{a}_{1}\right)\left(\tilde{a}_{N} \tilde{a}_{0}\right)=0$.

Remark 7.3 How should one visualize the action of $\varepsilon_{m}\left(\mathrm{n}_{\mathrm{TL}_{N}}\right) \subset \mathrm{n} \widehat{\mathrm{TL}}_{N+1}$ on the particle configurations on a circle with $N+1$ positions? Except for $a_{m}$, all generators of $\mathrm{nTL}_{N}$ are mapped to corresponding generators of $\mathrm{ntL}_{N+1}$. They will act as before, by moving a particle one step clockwise around the circle. Since $a_{m}$ is mapped by $\varepsilon_{m}$ to the product $\tilde{a}_{m+1} \tilde{a}_{m}$ in $\mathrm{nTL}_{N+1}$, it will move a particle from $m$ to $m+2$ as depicted in Fig. 5. In other words, the elements in $\varepsilon_{m}\left(\mathrm{nTL}_{N}\right)$ do not move a particle to or from position $m+1$.

Next we introduce a basis of $\widehat{n T L}_{N}$ that will enable us to see directly that these homomorphisms are embeddings. The basis has a simple description in terms of the graphical representation $\mathrm{V}$ from Sect. 4. For any two particle configurations with $1 \leq k \leq N-1$ particles corresponding to the increasing sequences $\mathbf{I}=\left\{1 \leq i_{1}<\cdots<i_{k} \leq N\right\}$ and $\mathbf{J}=\left\{1 \leq j_{1}<\cdots<j_{k} \leq N\right\}$, there is a monomial in $\mathrm{n \widehat {TL }}_{N}$ moving particles at the positions $\mathbf{J}$ to the positions $\mathbf{I}$. We require that every particle from $\mathbf{J}$ be moved by at least one step, but we do not prescribe explicitly which of the $j$ 's is mapped to which of the $i$ 's. For $\mathbf{I} \neq \mathbf{J}$, take $e_{\mathbf{I J}}$ to be the monomial such that the power of $q$ in $e_{\mathbf{I J}} v(\mathbf{J})= \pm q^{\ell} v(\mathbf{I})$ is minimal (under the assumption that every particle from $\mathbf{J}$ must be moved). By faithfulness of the graphical representation, $e_{\mathbf{I J}}$ is uniquely determined. For $\mathbf{I}=\mathbf{J}$, we have $e_{\mathbf{I I}}=a(\hat{\mathbf{I}})$, the special monomial defined in Sect. 4 , hence $e_{\mathbf{I I}} v(\mathbf{I})= \pm q v(\mathbf{I})$. Observe that one can write $\mathbf{t}_{k}=\sum_{|\mathbf{I}|=k} e_{\mathbf{I I}}$, where the sum runs over all possible increasing sequences $\mathbf{I}$ of length $k$, and that $\mathbf{t}_{k}^{\ell} e_{\mathbf{I J}}$ is a monomial, since all but one summand vanish for $k=|\mathbf{I}|$.

Remark 7.4 The condition that $e_{\mathbf{I J}}$ move all particles from $\mathbf{J}$ by at least one step guarantees that it acts as zero on all particle configurations with fewer particles than $|\mathbf{I}|=|\mathbf{J}|$.

For example, when $N=7$,

$$
e_{(2)(1)}=a_{1}, \quad e_{(0,2)(0,1)}=a_{6} a_{5} a_{4} a_{3} a_{1} a_{2} a_{0} a_{1} .
$$

(Note that $a_{1}$ moves $v(0,1)$ to $v(0,2)$, but this does not satisfy the requisite property that all the particles must be moved by at least one step.) If we apply the factorization of monomials from Theorem 6.1 to $e_{\mathbf{I J}}$, the minimality condition implies that $e_{\mathbf{I J}}=a_{\mathrm{fin}} \cdot 1 \cdot a\left(\underline{j}^{(0)}\right)$, where if $\mathbf{J}=\left\{j_{1}<\cdots<j_{k}\right\}$, then $\underline{j}^{(0)}$ is a sequence obtained by permuting the elements of $\mathbf{J}$.

Theorem 7.5 The set of monomials

$$
\{1\} \cup\left\{\boldsymbol{t}_{k}^{\ell} e_{\mathbf{I J}}\left|\ell \in \mathbb{Z}_{\geq 0}, 1 \leq\right| \mathbf{I}|=| \mathbf{J} \mid=k \leq N-1\right\}
$$


defines a $\mathbb{k}$-basis of the affine nilTemperley-Lieb algebra $n \widehat{T L}_{N}$.

Proof First, observe that $\mathbf{t}_{k}^{\ell} e_{\mathbf{I J}}$ is indeed a monomial since $|\mathbf{I}|=k$. We show that the elements $\mathbf{t}_{k}^{\ell} e_{\mathbf{I J}}$ act $\mathbb{k}$-linearly independently on the graphical representation $\mathbf{V}=$ $\bigoplus_{k=0}^{N}\left(\mathbb{k}[q] \otimes \bigwedge^{k} \mathbb{k}^{N}\right)$. By Remark 7.4, the monomial $e_{\mathbf{I J}}$ acts by zero on summands $\mathbb{k}[q] \otimes \bigwedge^{k^{\prime}} \mathbb{k}^{N}$ for $k^{\prime}<|\mathbf{I}|$. On $\mathbb{k}[q] \otimes \bigwedge^{|\mathbf{I}|} \mathbb{k}^{N}$, the matrix representing the action of $\mathbf{t}_{k}^{\ell} e_{\mathbf{I J}}$ relative to the standard basis has exactly one nonzero entry, and this one distinguishes all monomials with the same minimal number of particles $|\mathbf{I}|=|\mathbf{J}|$. From these two observations, the linear independence follows. On the other hand, given any nonzero monomial in $\mathrm{nTL}_{N}$, there exists a minimal particle configuration $\mathbf{J}$ on which it acts nontrivially. Recording the image particle configuration I and the power of $q$, we conclude that there is some $\ell$ so that the element $\mathbf{t}_{k}^{\ell} e_{\mathbf{I J}}$ acts on $\mathrm{V}$ in the same way as the given monomial does. Due to the faithfulness of this representation (see Theorem 4.5), the proposition follows.

In Sect. 8, a basis is constructed using a different approach (without relying on the faithful representation). Both bases are labelled by pairs of particle configurations (pairs of increasing sequences) together with a natural number $\ell$. Up to an index shift in the output configuration I and a shift of the natural number $\ell$, the labelling sets agree, and both bases actually coincide.

Proof (Theorem 7.1) We have already proven in Lemma 7.2 that $\varepsilon_{m}$ is an algebra homomorphism. Using Remark 7.3, observe that the monomial $e_{\mathbf{I J}} \in \mathrm{nTL}_{N}$ is mapped to a monomial $\tilde{e}_{\mathbf{I}^{\prime} \mathbf{J}^{\prime}} \in \mathrm{n} \widehat{\mathrm{TL}}_{N+1}$ (tilde again indicates in $\widehat{\mathrm{nL}}_{N+1}$ ), where the new index sets are obtained by $i \mapsto i$ for $0 \leq i \leq m$ and $i \mapsto i+1$ for $m+1 \leq i \leq N-1$. The injectivity follows since basis elements $\left(\sum_{|\mathrm{K}|=k} e_{\mathrm{KK}}\right)^{\ell} \cdot e_{\mathbf{I J}}$ of $\widehat{\mathrm{nL}}_{N}$ are mapped to basis elements $\left(\sum_{\left|\mathrm{K}^{\prime}\right|=k} \tilde{e}_{\mathrm{K}^{\prime} \mathrm{K}^{\prime}}\right)^{\ell} \cdot \tilde{e}_{\mathbf{I}^{\prime} \mathbf{J}^{\prime}}$ of $\widehat{n T L}_{N+1}$.

Remark 7.6 It is possible to verify this theorem on generators and relations in the language of Sect. 8 without using the graphical description.

Remark 7.7 Observe that these embeddings work specifically for the affine nilTemperleyLieb algebras but fail for the ordinary Temperley-Lieb algebras. The relation that fails to hold is the braid relation for Temperley-Lieb algebras, i.e. $a_{i} a_{i \pm 1} a_{i}=a_{i}$. Interestingly, the relation $a_{i}^{2}=\delta a_{i}$ is respected for $\delta=1$.

\section{A normal form and the faithfulness of the graphical representation}

In this section, we prove Theorem 4.5 which we recall here:

Proposition For $N \geq 3, V$ is a faithful $n \widehat{T L}_{N}$-module with respect to the action described in Definition 4.2.

For the proof, we will explicitly prove the linear independence of the matrices representing the monomials in $\widehat{n T L}_{N}$. We proceed in three steps: (1) First, we define a normal form for the monomials. (2) Next, we find a bijection between the monomials and certain pairs of particle configurations together with a power of $q$. In other words, we find a basis for $\mathrm{nTL}_{N}$ and describe a labeling set. (3) The final step is the description of the action of a monomial on $\mathrm{V}$ using its matrix realization. The matrices representing the monomials have a distinguished nonzero entry that is given in terms of the particle configurations and the power of $q$ from the bijection, and for most matrices, this is the only nonzero entry. From this description it will quickly follow that all these matrices are linearly independent. 


\subsection{Some useful facts}

The following lemma characterises nonzero monomials in $\mathrm{nTL}_{N}$. They correspond to fully commutative elements in $\widehat{\mathrm{TL}}_{N}$, see [9].

Lemma 8.1 The monomial $a(\underline{j}) \neq 0$ if and only if for any two neighbouring appearances of $a_{i}$ in $a(j)$ there are exactly one $a_{i+1}$ and one $a_{i-1}$ in between, apart from possible factors $a_{\ell}$ for $\ell \neq i-1, i, i+1$ (indices to be understood modulo $N$ ).

According to this result, two consecutive $a_{i}$ have to enclose $a_{i+1}$ and $a_{i-1}$, i.e. $a_{i} \ldots a_{i \pm 1} \ldots a_{i \mp 1} \ldots a_{i}$, with the dots being possible products of $a_{\ell}$ 's with $\ell \neq i \pm 1, i$. This lemma is a special case of $[9$, Lem. 2.6]; here is a quick proof for the convenience of the reader.

Proof The monomial $a(\underline{j})$ is zero if and only if we can bring two neighbouring factors $a_{i}$ together so that we obtain either $a_{i}^{2}$ ('square') or $a_{i} a_{i \pm 1} a_{i}$ ('braid'). But expressions of the form $a_{i} \ldots a_{i \pm 1} \ldots a_{i \mp 1} \ldots a_{i}$ cannot be resolved this way by commutativity relations. On the other hand, if there are two neighbouring factors $a_{i}$ with either none or only one of the terms $a_{i \pm 1}$ in between, we get after commutations either $a_{i}^{2}$ or $a_{i} a_{i \pm 1} a_{i}$. If there are at least two factors $a_{i+1}$ ( or $a_{i-1}$ ) in between the two $a_{i}$, one can repeat the argument: Either we can create a square or a braid, or we have at least two factors of the same kind in between. In the case of a square or a braid we are done; otherwise we pick two neighbouring $a_{i+k}$ in the $k$ th step of the argument. Since we always consider the space in between two neighbouring factors $a_{i}, a_{i+1}, \ldots, a_{i+k}$, none of the previous $a_{i}, a_{i+1}, \ldots, a_{i+k-1}$ occurs between the two neighbouring $a_{i+k}$. Unless we found a square or a braid in an earlier step, we end up in step $N-1$ with a subexpression of the form $a_{r} a_{r \pm 1}^{m} a_{r}$ which is zero for any $m \geq 0$.

Definition 8.2 For any $i \in\{0,1, \ldots, N-1\}$, we define a (clockwise) order $\stackrel{i}{\prec}$ on the set $\{0,1, \ldots, N-1\}$ starting at $i$ by

$$
i \stackrel{i}{\prec} i+1 \stackrel{i}{\prec} \ldots \stackrel{i}{\prec} i+N-1
$$

\subsection{Step 1: A normal form}

Given an arbitrary nonzero monomial $a(\underline{j})$ in $\widehat{n T L}_{N}$, reorder its factors according to the following algorithm (as usual, the indices are considered modulo $N$ ):

1. Find all factors $a_{i}$ in $a(j)$ with no $a_{i-1}$ to their right. We denote them by $a_{i_{1}}, \ldots, a_{i_{k}}$, ordered according to their appearance in $a(\underline{j})$; in other words, $a(\underline{j})$ is of the form

$$
a(\underline{j})=\ldots a_{i_{1}} \ldots a_{i_{2}} \ldots \ldots a_{i_{k}} .
$$


2. Move the $a_{i_{1}}, \ldots, a_{i_{k}}$ to the far right, without changing their internal order,

$$
a(\underline{j})=a\left(\underline{j^{\prime}}\right) \cdot\left(a_{i_{1}} a_{i_{2}} \ldots a_{i_{k}}\right)=a\left(\underline{j^{\prime}}\right) \cdot a\left(\underline{j}^{(0)}\right)
$$

for $j^{(0)}=\left(i_{1}, \ldots, i_{k}\right)$ and some sequence $j^{\prime}=\left(j\right.$ with $i_{1}, \ldots, i_{k}$ removed $)$. This is possible because

(a) by assumption, there is no $a_{i-1}$ to the right of an $a_{i}$ in this list;

(b) if for some $i, a_{i+1}$ occurs to the right of some $a_{i}$, then either $a_{i} \ldots a_{i+1} \ldots a_{i}$ would occur as a subword without $a_{i-1}$ in between, hence $a(j)=0$, or else $a_{i+1}$ does not have $a_{i}$ to its right, so it is one of the $a_{i_{1}}, \ldots, a_{i_{k}}$ itself, and will be moved to the far right of $a(j)$, too;

(c) $a_{i}$ commutes with all $a_{\ell}$ for $\ell \neq i-1, i+1$.

3. Repeat for $a\left(j^{\prime}\right)$ until we get

$$
a(\underline{j})=a\left(\underline{j}^{(m)}\right) \cdot a\left(\underline{j}^{(m-1)}\right) \cdots \cdots a\left(\underline{j}^{(1)}\right) \cdot a\left(\underline{j}^{(0)}\right)
$$

for sequences $\underline{j}^{(m)}, \ldots, \underline{j}^{(1)}$ obtained successively the same way as described above. Notice:

- Inside a sequence $j^{(n)}$, every index occurs at most once. If two consecutive indices occur within $\underline{j}^{(n)}$, they are increasingly ordered using the order $\stackrel{i_{k}}{\prec}$ from Definition 8.2 .

- For two consecutive sequences $j^{(n+1)}, j^{(n)}$ and for every index $i_{r}^{(n+1)}$ occurring in $\underline{j}^{(n+1)}$, we can find some index $i_{s}^{(n)}$ in $\underline{j}^{(n)}$ such that $i_{r}^{(n+1)}=i_{s}^{(n)}+1$.

- From that property, it also follows that the length of $\underline{j}^{(n+1)}$ is less or equal than the length of $\underline{j}^{(n)}$.

4. Reorder the factors $a\left(\underline{j}^{(m)}\right), \ldots, a\left(\underline{j}^{(1)}\right), a\left(\underline{j}^{(0)}\right)$ internally:

(a) Start with $a\left(j^{(0)}\right)$. There is some $0 \leq \hat{\imath} \leq N-1$ which does not occur in $j^{(0)}$, but $\hat{\imath}-1$ occurs. For example, this is satisfied by $\hat{\imath}=i_{k}+1$, as $i_{k}$ occurs in $j^{(0)}$ and is to the right of every other factor of $a(j)$. Choose the largest such $\hat{\imath}$ (with respect to the usual order). Then we can move $\hat{\imath}-1$ to the very right of the sequence $\underline{j}^{(0)}$, because $\hat{\imath}$ is not present, and $\hat{\imath}-2$ may only occur to the left of $\hat{\imath}-1$ due to the construction of $\underline{j}^{(0)}$. We proceed in the same way with those indices $\hat{\imath}-2, \hat{\imath}-3, \ldots, \hat{\imath}-(N-1)$ that appear in $\underline{j}^{(0)}$. The result is a reordering of the sequence $\underline{j}^{(0)}$ so that it is increasing from left to right with respect to $\stackrel{\hat{\imath}}{\prec}$.

(b) Repeat with all other factors $a\left(j^{(1)}\right), a\left(j^{(2)}\right), \ldots, a\left(j^{(m)}\right)$ taking as the initial righthand index of the sequence $\hat{\imath}, \hat{\imath} \overline{+} 1, \ldots, \overline{\hat{l}}+m-1$ respectively, and reordering within each $a\left(j^{(n)}\right)$ so that the indices are increasing from left to right with respect to $\stackrel{\hat{\imath}+n}{\prec}$. Throughout, the index $\hat{\imath}$ is the one from step (4a). 
Example 8.3 As an example for $\widehat{n T L}_{7}$, suppose $a(\underline{j})=a(64213542061325)$. (We omit the commas to simplify the notation.)

Find all $a_{i}$ without $a_{i-1}$ to their right:

Move them to the far right, and do not change their internal order: Repeat:

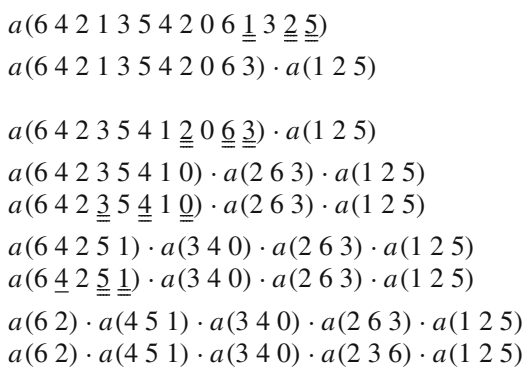

With the right-hand indices of the $a\left(\underline{j}^{(n)}\right), n \geq 0$, arranged according to $\hat{\imath}+m-1 \stackrel{\hat{\imath}}{\succ} \ldots \stackrel{\hat{\imath}}{\succ} \hat{\imath}+1 \stackrel{\hat{\imath}}{\succ} \hat{\imath}=6$ from left to right, reorder the factors in each $a\left(\underline{j}^{(n)}\right)$ increasingly with respect to $\stackrel{\hat{\imath}+n}{\prec}$ from left to right:

As a shorthand notation, in the following we often identify the index sequence $j$ with $a(j)$ (and manipulate $j$ according to the same relations as $a(j))$ as demonstrated in the following example.

Example 8.4 Let $N=6$.

$$
\begin{aligned}
(51230415023145023142) & =(1)(502)(3451)(2340)(1235)(0124) \\
& =\left(\begin{array}{llllllllll}
1 & 5 & 02 & 3451 & 2340 & 1235 & 0 & 124
\end{array}\right) .
\end{aligned}
$$

Lemma 8.5 Let a $(j)$ be a nonzero monomial in $n \widehat{T L}_{N}$, where we use as always the notation from Section 2. Let $a\left(\underline{j}^{(m)}\right), a\left(\underline{j}^{(m-1)}\right), \ldots, a\left(\underline{j}^{(1)}\right), a\left(\underline{j}^{(0)}\right)$ be the monomials constructed by the algorithm above.

1. The equality $a(\underline{j})=a\left(\underline{j}^{(m)}\right) a\left(\underline{j}^{(m-1)}\right) \cdots a\left(\underline{j}^{(1)}\right) a\left(\underline{j}^{(0)}\right)$ holds in $n \widehat{T L}_{N}$.

2. Given any two representatives $\bar{a}(j), a\left(j^{\#}\right)$ of the same element in $n \widehat{T L}_{N}$, the above algorithm creates the same representative $a\left(\underline{j}^{(m)}\right) a\left(\underline{j}^{(m-1)}\right) \cdots a\left(\underline{j}^{(1)}\right) a\left(\underline{j}^{(0)}\right)$ for both $a(\underline{j})$ and $a\left(\underline{j^{\#}}\right)$.

Proof 1. The algorithm never interchanges the order of two factors $a_{i}, a_{i \pm 1}$ with consecutive indices within $a(j)$. Hence, the reordering of the factors of $a(j)$ uses only the commutativity relation $\bar{a}_{i} a_{j}=a_{j} a_{i}$ for $i-j \neq \pm 1 \bmod N$ of $\widehat{\mathrm{TL}}_{N}$.

2. Two monomials $a(\underline{j}), a\left(\underline{j}^{\#}\right)$ in $\mathrm{nTL}_{N}$ are equal if and only if they only differ by applications of commutativity relations $a_{i} a_{j}=a_{j} a_{i}$ for $i-j \neq \pm 1 \bmod N$, hence, if and only if they contain the same number of factors $a_{i}$ for each $i$ and the relative position of each $a_{i}$ and $a_{i \pm 1}$ is the same. Since the outcome of the algorithm depends only on the relative positions of consecutive indices, the resulting decomposition $a\left(\underline{j}^{(m)}\right) a\left(\underline{j}^{(m-1)}\right) \cdots a\left(\underline{j}^{(1)}\right) a\left(\underline{j}^{(0)}\right)$ is the same. 
We have shown the following. In stating this result and subsequently, whenever we refer to monomials in normal form, we assume the monomial is nonzero and nonconstant, in particular the sequence $j$ is nonempty.

Theorem 8.6 Assume $N \geq 3$.

1. The algorithm in Step 1 above provides a normal form for nonzero monomials a $(j)$ in the generators $a_{i}$ of $n \widehat{T L}_{N}$, or equivalently for nonzero fully commutative monomials in $\widehat{\mathrm{TL}}_{N}$, so that

$a(\underline{j})=\left(a_{i_{1}}^{(m)} \ldots a_{i_{k}}^{(m)}\right) \ldots\left(a_{i_{1}}^{(n+1)} \ldots a_{i_{k}}^{(n+1)}\right)\left(a_{i_{1}}^{(n)} \ldots a_{i_{k}}^{(n)}\right) \ldots\left(a_{i_{1}}^{(1)} \ldots a_{i_{k}}^{(1)}\right)\left(a_{i_{1}} \ldots a_{i_{k}}\right)$, where $a_{i_{\ell}}^{(n)} \in\left\{1, a_{0}, a_{1}, \ldots, a_{N-1}\right\}$ for all $1 \leq n \leq m, 1 \leq \ell \leq k$, and

$$
a_{i_{\ell}}^{(n+1)} \in \begin{cases}\{1\} & \text { if } a_{i_{\ell}}^{(n)}=1, \\ \left\{1, a_{j+1}\right\} & \text { if } a_{i_{\ell}}^{(n)}=a_{j} .\end{cases}
$$

The factors $a_{i_{1}}, \ldots, a_{i_{k}}$ are determined by the property that the generator $a_{i_{\ell}-1}$ does not appear to the right of $a_{i_{\ell}}$ in the original presentation of the monomial. The internal ordering of the factors is increasing with respect to the relation $\stackrel{\hat{\imath}}{\succ}$, as in Step (4a) of the normal form algorithm, where $\hat{\imath}$ is the largest value in $\{0,1, \ldots, N-1\}$ such that $\hat{\imath}-1 \notin\left\{i_{1}, \ldots, i_{k}\right\}$, but $\hat{\imath} \in\left\{i_{1}, \ldots, i_{k}\right\}$.

2. The set $\{a(\underline{j})$ in normal form $\} \cup\{1\}$ is a $\mathbb{k}$-basis of $n \widehat{T L}_{N}$.

\subsection{Step 2: Labelling of basis elements}

Definition 8.7 Given $a(\underline{j})=a\left(\underline{j}^{(m)}\right) a\left(\underline{j}^{(m-1)}\right) \cdots a\left(\underline{j}^{(1)}\right) a\left(\underline{j}^{(0)}\right)$ in normal form, we call $j^{(\ell)}$ the $\ell$ th block of $j$, and a string of indices of maximal length of the form $i_{s} \in j^{(0)}, i_{s}+1 \in$ $\bar{j}^{(1)}, i_{s}+2 \in \underline{j}^{(2)}, \ldots$ (modulo $N$ ) the $s$ th strand of $\underline{j}$. We use the notation $\left[\ldots, i_{s}+1, i_{s}\right]$ for the strands.

Example 8.8 Let $N=6$, and consider Example 8.4 once again, where

$$
\underline{j}=\left(\begin{array}{llllllllllllll}
1 & 5 & 0 & 3 & 3451 & 2340 & 1235 & 0 & 124
\end{array}\right) .
$$

The blocks are $\underline{j}^{(0)}=(0124), \underline{j}^{(1)}=(1235), \underline{j}^{(2)}=(2340), \underline{j}^{(3)}=(3451), \underline{j}^{(4)}=(502)$, and $j^{(5)}=(1)$. The strands are [3210], [54321], [105432] and [21054]. In particular, strands (and blocks) can have different lengths, but the longest strand has length $m=6$.

Each monomial $a(\underline{j}) \in \mathrm{nTL}_{N}$ determines two sets $\mathbf{I}_{\underline{j}}^{\text {in }}, \mathbf{I}_{\underline{j}}^{\text {out }}$ and an integer $\ell_{\underline{j}} \in \mathbb{Z}_{\geq 0}$ as follows:

$$
\begin{aligned}
\mathbf{I}_{\underline{j}}^{\text {in }} & =\{i \in\{0,1, \ldots, N-1\} \mid \text { no } i-1 \text { to the right of } i \text { in } \underline{j}\} \\
\mathbf{I}_{\underline{j}}^{\text {out }} & =\{i \in\{0,1, \ldots, N-1\} \mid \text { no } i+1 \text { to the left of } i \text { in } \underline{j}\} \\
\ell_{\underline{j}} & =\text { the number of zeros in } \underline{j} .
\end{aligned}
$$

These are well defined because, as in the proof of Lemma 8.5, any element of $n \widehat{T L}_{N}$ is uniquely determined by the number of factors $a_{i}$ and the relative position of each $a_{i}$ and $a_{i \pm 1}$, for all $i$. The set $\mathbf{I}_{j}^{\text {in }}$ equals the underlying set of $\underline{j}^{(0)}$ in the normal form from the 
algorithm above. All strands of $\underline{j}$ begin with an element in $\mathbf{I}_{\underline{j}}^{\text {in }}$ and end with an element from $\mathbf{I}_{j}^{\text {out }}$.

The goal of this subsection is to show

\section{Proposition 8.9 The mapping}

$$
\begin{aligned}
\psi:\left\{a(\underline{j}) \in n \widehat{T L}_{N} \text { in normal form }\right\} & \rightarrow \mathcal{P}_{N} \times \mathcal{P}_{N} \times \mathbb{Z}_{\geq 0} \\
a(\underline{j}) & \mapsto\left(\mathbf{I}_{\underline{j}}^{\text {in }}, \mathbf{I}_{\underline{j}}^{\text {out }}, \ell_{\underline{j}}\right),
\end{aligned}
$$

is injective, where $\mathcal{P}_{N}$ is the power set of $\{0,1, \ldots, N-1\}$.

Remark 8.10 The map $\psi$ is defined so that in the graphical description of the representation $\mathrm{V}$ of $\mathrm{nTL}_{N}$, the set $\mathrm{I}_{j}^{\text {in }}$ equals the set of positions where $a(\underline{j})$ expects a particle to be. The set $\mathbf{I}_{j}^{\text {out }}$ equals the set of positions where $a(\underline{j})$ moves the particles from $\mathbf{I}_{j}^{\text {in }}$, but each one is translated by 1 , that is,

$$
a(\underline{j}) \text { applied to a particle at } i \in \mathbf{I}_{\underline{j}}^{\text {in }} \text { gives a particle at } j+1 \text { for some } j \in \mathbf{I}_{\underline{j}}^{\text {out }} .
$$

The map $\psi$ is far from being surjective. An obvious constraint is that $\left|\mathbf{I}_{\underline{j}}^{\mathrm{in}}\right|=\left|\mathbf{I}_{\underline{j}}^{\text {out }}\right|$, and furthermore, for some pairs ( $\left.\mathbf{I}_{j}^{\text {in }}, \mathbf{I}_{j}^{\text {out }}\right)$, one can only obtain sufficiently large values $\bar{\ell}_{\underline{j}}$.

To ease the presentation, we start by proving injectivity of the restriction $\psi_{0}$ of $\psi$ to those monomials $a(j)$ in normal form whose first element $i_{1}$ of $\underline{j}^{(0)}$ is 0 . The proof itself will amount to counting indices.

Proposition 8.11 The map

$$
\begin{aligned}
\psi_{0}:\left\{a(\underline{j}) \in n \widehat{T L}_{N} \text { in normal form, with } i_{1}=0\right\} \rightarrow \mathcal{P}_{N} \times \mathcal{P}_{N} \times \mathbb{Z}_{\geq 0}, \\
\quad a(\underline{j}) \mapsto\left(\mathbf{I}_{\underline{j}}^{\text {in }}, \mathbf{I}_{\underline{j}}^{\text {out }}, \ell_{\underline{j}}\right)
\end{aligned}
$$

is injective.

Before beginning the proof of this result, we note that for monomials $a(j)$ with $i_{1}=0$, the inequality $i_{k}<N-1$ must hold in $\mathbf{I}_{\underline{j}}^{\text {in }}$, since $i_{1}=0$ implies that $i_{1}-1=N-1$ is not an element of $\mathbf{I}_{\underline{j}}^{\text {in }}$. Consequently, the ordering of the indices in $\mathbf{I}_{\underline{j}}^{\text {in }}$ agrees with the natural ordering of $\mathbb{Z}$, so we can regard $\left(\mathbf{I}_{\underline{j}}^{\text {in }},<\right)$ as a subset of $(\mathbb{Z},<)$ and replace the modular index sequence $\underline{j}$ by an integral index sequence $\underline{j}^{\mathbb{Z}}$ such that $\underline{j}^{\mathbb{Z}}(\bmod N)=\underline{j}$ as follows:

Definition 8.12 Assume $\underline{j}=\underline{j}^{(m)} \cdots \underline{j}^{(1)} \cdot \underline{j}^{(0)}$ is a normal form sequence with $\underline{j}^{(0)}=$ $\left\{0=i_{1}<\cdots<i_{k}<N-1\right\}$ and $\underline{j}^{(n)}=\left(i_{h_{1}}+n, \ldots, i_{h_{k(n)}}+n\right) \subseteq\left(i_{1}+n, \ldots, i_{k}+n\right)$, where indices in $j^{(n)}$ are modulo $\bar{N}$ and $1 \leq k(n) \leq k$ for all $1 \leq n \leq m$. The integral normal form sequence for $j$ is

$$
\underline{j}^{\mathbb{Z}}=\left(\underline{j}^{(m)}\right)^{\mathbb{Z}} \cdots \cdot\left(\underline{j}^{(1)}\right)^{\mathbb{Z}} \cdot \underline{j}^{(0)} \quad \text { where } \quad\left(\underline{j}^{(n)}\right)^{\mathbb{Z}}:=\left(i_{h_{1}}+n, \ldots, i_{h_{k(n)}}+n\right) \in \mathbb{Z}^{k(n)}
$$

for $n=1, \ldots, m$.

Example 8.13 We continue Example 8.4 with $N=6$.

$$
\begin{aligned}
& \text { If } \underline{j}=\left(\begin{array}{lllllllll}
1 & 50 & 2 & 3451 & 2340 & 1235 & 0 & 124
\end{array}\right) \text {, } \\
& \text { then } \underline{j}^{\mathbb{Z}}=\left(\begin{array}{lllllll}
7 & 568 & 3457 & 2346 & 1235 & 0 & 124
\end{array}\right) \text {. }
\end{aligned}
$$


Our proof of Proposition 8.11 will hinge upon the following technical lemma.

Lemma 8.14 Let $\underline{j} \mathbb{Z}$ be the integral normal form sequence for $\underline{j}$ and let $\left[i_{s}, \ldots, i_{s}+n_{s}\right]$ for $s=1, \ldots, k$ be the strands of $\underline{j}^{\mathbb{Z}}$. Assume $i_{1}=0$. Then

(a) $n_{1}=i_{1}+n_{1}<i_{2}+n_{2}<\cdots<i_{k}+n_{k}$;

(b) $i_{k}+n_{k}<i_{1}+n_{1}+N=n_{1}+N$.

We postpone the proof of this result and proceed directly to proving the proposition.

Proof (Proposition 8.11) Since the sequence $j$ will be fixed throughout the proof, we will drop the subscript $\underline{j}$ on $\mathbf{I}_{j}^{\text {in }} \mathbf{I}_{j}^{\text {out }}, \ell_{j}$. To show the injectivity of $\psi_{0}$, we consider the factorization $\psi_{0}=\gamma \circ \beta \circ \alpha$ given by

$$
\psi_{0}: a(\underline{j}) \stackrel{\alpha}{\longmapsto} a\left(\underline{j}^{\mathbb{Z}}\right) \stackrel{\beta}{\longmapsto}\left(\left(\mathbf{I}^{\text {in }}\right)^{\mathbb{Z}},\left(\mathbf{I}^{\text {out }}\right)^{\mathbb{Z}}\right) \stackrel{\gamma}{\longmapsto}\left(\mathbf{I}^{\text {in }}, \mathbf{I}^{\text {out }}, \ell\right),
$$

where $\left(\mathbf{I}^{\text {in }}\right)^{\mathbb{Z}}=\mathbf{I}^{\text {in }}$ and $\left(\mathbf{I}^{\text {out }}\right)^{\mathbb{Z}}=\left\{i \in \underline{j}^{\mathbb{Z}} \mid\right.$ no $i+1$ to the left of $\left.i\right\}$ similar to the definition of $\mathbf{I}^{\text {out }}$. The map $\alpha$ replaces indices in $\mathbb{Z} / N \mathbb{Z}$ by indices in $\mathbb{Z}$ as in Definition 8.12 above. The map $\beta$ is given by reading off $\left(\mathbf{I}^{\text {out }}\right)^{\mathbb{Z}}$ and $\left(\mathbf{I}^{\text {in }}\right)^{\mathbb{Z}}$ from $\underline{j}^{\mathbb{Z}}$. The map $\gamma$ sends the pair $\left(\left(\mathbf{I}^{\text {in }}\right)^{\mathbb{Z}},\left(\mathbf{I}^{\text {out }}\right)^{\mathbb{Z}}\right)$ to a triple consisting of the respective images $\mathbf{I}^{\text {in }}, \mathbf{I}^{\text {out }}$ modulo $N$ of the pair and the integer $\ell=1+\sum \ell_{r}$ where $\ell_{r}=\left\lfloor\frac{j_{r}}{N}\right\rfloor$ for each $j_{r} \in\left(\mathbf{I}^{\text {out }}\right)^{\mathbb{Z}}$. The summand 1 corresponds to $0=i_{1}$; all other occurrences of 0 are counted by $\sum \ell_{r}$. Now we check injectivity.

The map $\alpha$ is clearly injective since $j^{\mathbb{Z}} \mapsto j^{\mathbb{Z}}(\bmod N)$ is a left inverse map.

To see that $\beta$ is injective, we need to know that $\underline{j}^{\mathbb{Z}}$ can be uniquely reconstructed from $\left(\left(\mathbf{I}^{\text {in }}\right)^{\mathbb{Z}},\left(\mathbf{I}^{\text {out }}\right)^{\mathbb{Z}}\right)$. Observe that $j^{\mathbb{Z}}$ is determined by knowing all the 'strands' $i_{s}, i_{s}+1, i_{s}+$ $2, \ldots, i_{s}+n_{s}$ for $1 \leq s \leq k$, hence by assigning an element $i_{s}+n_{s} \in\left(\mathbf{I}^{\text {out }}\right)^{\mathbb{Z}}$ to each $i_{s} \in\left(\mathrm{I}^{\mathrm{in}}\right)^{\mathbb{Z}}$. But it follows from Lemma 8.14 (a) that $i_{1}+n_{1}$ must be the smallest element of $\left(\mathbf{I}^{\text {out }}\right)^{\mathbb{Z}}, i_{2}+n_{2}$ the second smallest, etc., so that the element $i_{s}+n_{s}$ is assigned to the $s$ th element in $\mathbf{I}^{\text {in }}$, that is, to $i_{s}$.

Now to see that $\gamma$ is injective, we need to recover $\left(\left(\mathbf{I}^{\text {in }}\right)^{\mathbb{Z}},\left(\mathbf{I}^{\text {out }}\right)^{\mathbb{Z}}\right)$ in a unique way from $\left(\mathbf{I}^{\text {in }}, \mathbf{I}^{\text {out }}, \ell\right)$. Write $\mathbf{I}^{\text {in }}=\left\{0=i_{1}<\cdots<i_{k}<N-1\right\}$, and set $\left(\mathbf{I}^{\text {in }}\right)^{\mathbb{Z}}:=\mathbf{I}^{\text {in }}$. By Lemma 8.14 (a), we know that ( $\left.\mathbf{I}^{\text {out }}\right)^{\mathbb{Z}}$ is of the form $\left(i_{1}+n_{1}<\cdots<i_{k}+n_{k}\right)$, and since the elements of $\mathbf{I}^{\text {out }}$ have to be equal to the elements of $\left(\mathbf{I}^{\text {out }}\right)^{\mathbb{Z}}$ modulo $N$, we can write $i_{r}+n_{r}=N \ell_{r}+d_{r}$ for $\ell_{r}=\left\lfloor\frac{i_{r}+n_{r}}{N}\right\rfloor$ and some $d_{r} \in \mathbf{I}$ out . Comparing $\ell_{r}$ and $\ell_{s}$ for $r<s$, we have

$$
N \ell_{r} \leq N \ell_{r}+d_{r}=i_{r}+n_{r}<i_{s}+n_{s}=N \ell_{s}+d_{s} \leq N\left(\ell_{s}+1\right) .
$$

So $\ell_{r}<\ell_{s}+1$, i.e. $\ell_{r} \leq \ell_{s}$. Similarly, we obtain from (b) of Lemma 8.14 that $\ell_{k} \leq \ell_{1}+1$. As a result,

$$
N \ell_{k} \leq N \ell_{k}+d_{k}=i_{k}+n_{k}<i_{1}+n_{1}+N=N\left(\ell_{1}+1\right)+d_{1} \leq N\left(\ell_{1}+2\right),
$$

i.e. $\ell_{k}<\ell_{1}+2$. Together we have $\ell_{1}=\cdots=\ell_{s}<\ell_{s+1}=\cdots=\ell_{1}+1$ for some $1<s \leq k$ (where we treat the case $s=k$ by $\ell_{1}=\cdots=\ell_{k}$ ). Set $\tilde{\ell}:=\ell_{1}$. Then

$$
\begin{aligned}
& i_{r}+n_{r}=N \tilde{\ell}+d_{r} \quad \text { for } 1 \leq r \leq s, \\
& i_{r}+n_{r}=N(\tilde{\ell}+1)+d_{s} \text { for } s+1 \leq r \leq k .
\end{aligned}
$$

As a first consequence,

$$
\ell=1+\sum_{r} \ell_{r}=1+k \tilde{\ell}+(k-s)
$$


which determines $\tilde{\ell}=\left\lfloor\frac{\ell-1}{k}\right\rfloor$, and hence all $\ell_{r}$, as well as the index $s$. Using Lemma 8.14, we determine that

$$
i_{s+1}+n_{s+1}<\cdots<i_{k}+n_{k}<i_{1}+n_{1}+N<\cdots<i_{s}+n_{s}+N,
$$

and so

$$
N(\tilde{\ell}+1)+d_{s+1}<\cdots<N(\tilde{\ell}+1)+d_{k}<N(\tilde{\ell}+1)+d_{1}<\cdots<N(\tilde{\ell}+1)+d_{s} .
$$

Therefore, $d_{s+1}<\cdots<d_{k}<d_{1}<\cdots<d_{s}$, which fixes the choice of $d_{r}$ for all $r$. We conclude that given $\left(\mathbf{I}^{\text {in }}, \mathbf{I}^{\text {out }}, \ell\right.$ ), we can reconstruct $\left(\mathbf{I}^{\text {out }}\right)^{\mathbb{Z}}$ by setting $i_{r}+n_{r}:=N \ell_{r}+d_{r}$. This completes the proof of Proposition 8.11.

Proof (Lemma 8.14) (a) Let $j^{\mathbb{Z}}$ be a nonempty integral normal form sequence with $0=i_{1}<$ $\cdots<i_{k} \leq N-1$ and strands $\left[i_{r}, \ldots, i_{r}+n_{r}\right]$ for $1 \leq r \leq k$. Assume that there is some index $1 \leq t \leq k-1$ such that $i_{t}+n_{t} \geq i_{t+1}+n_{t+1}$. Since $i_{t}<i_{t+1}$, we have $n_{t}>n_{t+1}$. So

$$
\underline{j}^{\mathbb{Z}}=\ldots \underbrace{\left(\ldots i_{t}+n_{t} \ldots\right)}_{\text {the } n_{t} \text { th bracket }} \cdots \underbrace{\left(\ldots i_{t}+n_{t+1} i_{t+1}+n_{t+1} \ldots\right)}_{\text {the } n_{t+1} \text { th bracket }} \cdots
$$

From $i_{t}+n_{t+1}<i_{t+1}+n_{t+1} \leq i_{t}+n_{t}$ it follows that there is some integer $n_{t+1}<p \leq n_{t}$ such that $i_{t+1}+n_{t+1}=i_{t}+p$ appears in the strand $\left[i_{t}, \ldots, i_{t}+n_{t}\right]$, i.e.

$$
\underline{j}^{\mathbb{Z}}=\ldots \underbrace{\left(\ldots i_{t}+n_{t} \ldots\right)}_{\text {the } n_{t} \text { th bracket }} \cdots \underbrace{\left(\ldots i_{t}+p \ldots\right)}_{\text {the } p \text { th bracket }} \cdots \underbrace{\left(\ldots i_{t}+n_{t+1} i_{t+1}+n_{t+1} \ldots\right)}_{\text {the } n_{t+1} \text { th bracket }} \cdots
$$

with $i_{t}+p=i_{t+1}+n_{t+1}$. But by the definition of the strands, there is no $i_{t+1}+n_{t+1}+1$ appearing to the left of $i_{t+1}+n_{t+1}$. Due to Lemma 8.1, we know that (even modulo $N$ ) there is no repetition of $i_{t+1}+n_{t+1}$ to the left. Thus $i_{t}+p=i_{t+1}+n_{t+1}$ is not possible, and we obtain $i_{1}+n_{1}<i_{2}+n_{2}<\cdots<i_{k}+n_{k}$.

For (b) of Lemma 8.14, assume $i_{k}+n_{k} \geq i_{1}+n_{1}+N$. It is true generally that $N>i_{k}$, so we get $i_{k}+n_{k} \geq i_{1}+n_{1}+N>i_{k}+n_{1}$. Hence $i_{1}+n_{1}+N=i_{k}+b$ for some $n_{1}<b \leq n_{k}$, i.e. $i_{1}+n_{1}+N$ appears in the strand $\left[i_{k}, \ldots, i_{k}+n_{k}\right]$ and we have

$$
\underline{j}^{\mathbb{Z}}=\ldots \underbrace{\left(\ldots i_{k}+n_{k}\right)}_{\text {the } n_{k} \text { th bracket }} \cdots \underbrace{\left(\ldots i_{k}+b \ldots\right)}_{\text {the } b \text { th bracket }} \cdots \underbrace{\left(i_{1}+n_{1} \ldots i_{k}+n_{1}\right)}_{\text {the } n_{1} \text { th bracket }} \cdots
$$

Here it may be that the $n_{k}$ th bracket and the $b$ th bracket coincide, but in any case, we find that $i_{k}+b=i_{1}+n_{1}+N=i_{1}+n_{1} \bmod N$, and so $i_{k}+b$ appears to the left of $i_{1}+n_{1}$. By the definition of the strands, there is no $i_{1}+n_{1}+1$ to the left of $i_{1}+n_{1}$, and from Lemma 8.1 we deduce that in $j=j^{\mathbb{Z}} \bmod N$ there is no $i_{1}+n_{1} \bmod N$ to the left of $i_{1}+n_{1}$ allowed, which leads to a contradiction. Hence $i_{k}+n_{k}<i_{1}+n_{1}+N$ must hold.

Having established that $\psi$ is injective when restricted to sequences with $i_{1}=0$, we now show the injectivity of $\psi$ in general. 
Proof (Proposition 8.9) We have the following disjoint decompositions according to the smallest value $i_{1}$ in $\underline{j}^{(0)}$ for $\underline{j}$ :

$$
\begin{aligned}
\{a(\underline{j}) \text { in normal form }\} & =\coprod_{i}\left\{a(\underline{j}) \text { in normal form, with } i_{1}=i\right\} \\
\left\{\left(\mathbf{I}_{\underline{j}}^{\text {in }}, \mathbf{I}_{\underline{j}}^{\text {out }}, \ell_{\underline{j}}\right)\right\}= & \coprod_{i}\left\{\left(\mathbf{I}_{\underline{j}}^{\text {in }}, \mathbf{I}_{\underline{j}}^{\text {out }}, \ell_{\underline{j}}\right) \mid i_{1}=i \in \mathbf{I}_{\underline{j}}^{\text {in }}\right\} \\
\psi= & \coprod_{i}\left(\psi_{i}:\left\{a(\underline{j}) \text { in normal form, with } i_{1}=i\right\}\right. \\
& \left.\rightarrow\left\{\left(\mathbf{I}_{\underline{j}}^{\text {in }}, \mathbf{I}_{\underline{j}}^{\text {out }}, \ell_{\underline{j}}\right) \mid i_{1}=i \in \mathbf{I}_{\underline{j}}^{\text {in }}\right\}\right) .
\end{aligned}
$$

By Proposition 8.11, the map $\psi_{0}: a(\underline{j}) \mapsto\left(\mathbf{I}_{j}^{\text {in }}, \mathbf{I}_{j}^{\text {out }}, \ell_{\underline{j}}\right)$ restricted to those $a(\underline{j})$ with $i_{1}=0$ is injective. We argue next that by an index shift this result is true for all other $\psi_{i}$.

Now it follows from Proposition 8.11 that the map

$\widehat{\psi}_{0}:\left\{a(\underline{j}) \in \mathrm{nTL}_{N}\right.$ in normal form, with $\left.i_{1}=0\right\} \rightarrow\left\{\left(\mathbf{I}_{\underline{j}}^{\text {in }}, \mathbf{I}_{\underline{j}}^{\text {out }}, \widehat{\ell_{\underline{j}}}\right) \mid i_{1}=0 \in \mathbf{I}_{\text {in }}\right\}$ is injective, where $\widehat{\ell_{\underline{j}}}$ counts the occurences of $N-i$ in $\underline{j}$. Recall that $\ell_{\underline{j}}=\sum_{r} \ell_{r}+1$ and $\ell_{r}$ is the number of zeros in the $r$ th strand $\left[i_{r}, \ldots, i_{r}+n_{r}\right]$ of $\underline{j} \bmod N$. Now observe that we can obtain $\ell_{\underline{j}}$ from $\widehat{\ell_{\underline{j}}}$ as

$$
\ell_{\underline{j}}=\widehat{\ell}_{\underline{j}}-\left|\left\{d_{r} \in \mathbf{I}_{\underline{j}}^{\text {out }} \mid d_{r} \geq N-i\right\}\right|+\left|\left\{i_{r} \in \mathbf{I}_{\underline{j}}^{\text {in }} \mid i_{r}>N-i\right\}\right|+1,
$$

which follows from a computation using $\widehat{\ell_{\underline{j}}}=\sum_{r} \widehat{\ell_{r}}$ and

$$
\begin{aligned}
\widehat{\ell_{r}} & =\text { the number of } N-i \text { in the } r \text { th strand }\left[i_{r}, \ldots, i_{r}+n_{r}\right] \bmod N \\
& = \begin{cases}\left\lfloor\frac{i_{r}+n_{r}+i}{N}\right\rfloor & \text { if } i_{r} \leq N-i \\
\left\lfloor\frac{i_{r}+n_{r}+i}{N}\right\rfloor-1 & \text { if } i_{r}>N-i\end{cases} \\
& = \begin{cases}\left\lfloor\frac{N \ell_{r}+d_{r}+i}{N}\right\rfloor & \text { if } i_{r} \leq N-i \\
\left\lfloor\frac{N \ell_{r}+d_{r}+i}{N}\right\rfloor-1 & \text { if } i_{r}>N-i\end{cases} \\
& = \begin{cases}\ell_{r}+1 & \text { if } i_{r} \leq N-i \text { and } d_{r}+i \geq N \\
\ell_{r} & \text { if } i_{r} \leq N-i \text { and } d_{r}+i<N \\
\ell_{r} & \text { if } i_{r}>N-i \text { and } d_{r}+i \geq N \\
\ell_{r}-1 & \text { if } i_{r}>N-i \text { and } d_{r}+i<N .\end{cases}
\end{aligned}
$$

We obtain $\psi_{i}$ by first shifting the indices of $\underline{j}$ by subtracting $i$ from each index, $\underline{j}-$ $(i, \ldots, i)$, then applying $\widehat{\psi}_{0}$, and finally shifting the indices from $\mathbf{I}_{j}^{\text {in }}$ and $\mathbf{I}_{j}^{\text {out }}$ by adding $\bar{i}$ to each. Hence, $\psi_{i}$ is injective for each $i$, and $\psi$ is injective because the unions are disjoint.

\subsection{Step 3: Description and linear independence of the matrices}

Recall that the standard $\mathbb{k}$-basis of the representation $\mathrm{V}=\bigoplus_{k=0}^{N}\left(\mathbb{k}[q] \otimes \bigwedge^{k} \mathbb{k}^{N}\right)$ is given by

$$
\left\{q^{\ell} \cdot v_{i_{1}} \wedge \cdots \wedge v_{i_{k}} \mid \ell \in \mathbb{Z}_{\geq 0}, 1 \leq i_{1}<\cdots<i_{k} \leq N\right\}
$$


where $\left(i_{1}, \ldots, i_{k}\right)$ is identified with the particle configuration having particles in those positions in the graphical description. Now we describe with respect to this basis the matrix representing a nonzero monomial $a(j) \in \mathrm{nTL}_{N}$ as a $2^{N} \times 2^{N}$-matrix with entries in $\mathbb{k}[q]$. Since $\mathrm{V}$ decomposes as a $\widehat{n T L}_{N}$-module into submodules $\mathbb{k}[q] \otimes \bigwedge^{k} \mathbb{k}^{N}$ for $k=0,1, \ldots, N$, the matrix of $a(j)$ is block diagonal with $N+1$ blocks $A_{0}, A_{1}, \ldots, A_{N}$, where $A_{0}=A_{N}=(0)$ corresponding to the trivial representation.

$$
a(\underline{j})=\left(\begin{array}{ccccc}
0 & 0 & & \cdots & 0 \\
0 & A_{1} & & & \vdots \\
& & \ddots & & \\
\vdots & & & A_{N-1} & 0 \\
0 & \cdots & & 0 & 0
\end{array}\right)
$$

The block $A_{k}$ is a $\left(\begin{array}{l}N \\ k\end{array}\right) \times\left(\begin{array}{l}N \\ k\end{array}\right)$-matrix, with entries from $\mathbb{k}[q]$ indexed by all possible particle configurations whose number of particles equal to $k$.

Now fix a nonzero monomial $a(\underline{j})$ in normal form that is specified by the triple $\left(\mathbf{I}_{\underline{j}}^{\text {in }}, \mathbf{I}_{\underline{j}}^{\text {out }}, \ell_{\underline{j}}\right)$ defined in Step 2. Let $k=\left|\mathbf{I}_{\underline{j}}^{\text {in }}\right|$. All blocks $A_{1}, \ldots, A_{k-1}$ are zero since $a(\underline{j})$ expects at least $k$ particles. For $r>k$ there might be nonzero blocks $A_{r}$. Such nonzero blocks appear unless the particles from $\mathbf{I}_{\underline{j}}^{\text {in }}$ are moved around the whole circle with no position left out, in which case there are no surplus particles allowed. This occurs if $a(j)$ contains at least every other generator $a_{i}, a_{i+2}, \ldots$..

More importantly, the block $A_{k}$ has precisely one nonzero entry, and this is given by

$$
\left(A_{k}\right)_{\mathbf{I}_{\underline{j}}^{\text {in }}, \mathbf{I}_{\underline{j}}^{\text {out }}}= \pm q^{\ell_{\underline{j}}} .
$$

From this we see first that all matrices representing monomials $a(j)$ in normal form with $\left|\mathbf{I}_{j}^{\text {in }}\right|=N-1$ are $\mathbb{k}$-linearly independent: They have only one nonzero entry which is equal to $\pm q^{\ell_{j}}$ at position ( $\left.\mathbf{I}_{\underline{j}}^{\text {in }}, \mathbf{I}_{\underline{j}}^{\text {out }}\right)$. Furthermore, if all matrices representing monomials $a(\underline{j})$ in normal form with $\left|\mathbf{I}_{\underline{j}}^{\text {in }}\right| \geq k$ are $k$-linearly independent, then also all matrices representing monomials $a(\underline{j})$ in normal form with $\left|\mathbf{I}_{\underline{j}}^{\mathrm{in}}\right| \geq k-1$ are $\mathbb{k}$-linearly independent. This follows because the additional monomials $a(\underline{j})$ with $\left|\mathbf{I}_{j}^{\text {in }}\right|=k-1$ have nonzero entries $\left(A_{k-1}\right)_{\mathbf{I}_{\underline{j}}^{\text {in }}, \mathbf{I}_{\underline{j}}^{\text {out }}}= \pm q^{\ell_{\underline{j}}}$ in the $(k-1)$ th block which is zero for all $a(\underline{j})$ with $\left|\mathbf{I}_{\underline{j}}^{\text {in }}\right| \geq k$. So by induction, all matrices representing monomials $a(j)$ in normal form are $\mathbb{k}$-linearly independent. Since all of them have a zero entry in the upper left (and lower right) corner, we may add the identity matrix to the linearly independent set of matrices, and it remains linearly independent. So the representation of $\mathrm{nTL}_{N}$ on $\mathrm{V}$ is faithful, because according to Theorem 8.6, $\{a(j)$ in normal form $\} \cup\{1\}$ is a $\mathbb{k}$-basis of $n \widehat{\mathrm{TL}}_{N}$.

Section 8 has given a normal form for each monomial and has provided an alternate proof of the faithfulness of the representation of $\mathrm{nTL}_{N}$ by elementary arguments.

Acknowledgments Open access funding provided by Max Planck Society Max Planck Institute for Mathematics.

Open Access This article is distributed under the terms of the Creative Commons Attribution 4.0 International License (http://creativecommons.org/licenses/by/4.0/), which permits unrestricted use, distribution, and 
reproduction in any medium, provided you give appropriate credit to the original author(s) and the source, provide a link to the Creative Commons license, and indicate if changes were made.

\section{References}

1. Alharbat, S.: A classification of affine fully commutative elements. arXiv:1311.7089

2. Berenstein, A., Fomin, S., Zelevinsky, A.: Parametrizations of canonical bases and totally positive matrices. Adv. Math. 122(1), 49-149 (1996)

3. Bernštein, I.N., Gel'fand, I.M., Gel'fand, S.I.: Schubert cells, and the cohomology of the spaces $G / P$. Uspehi Mat. Nauk 28(3), 3-26 (1973)

4. Billey, S.C., Jockusch, W., Stanley, R.P.: Some combinatorial properties of Schubert polynomials. J. Algebraic Comb. 2(4), 345-374 (1993)

5. Brichard, J.: On Using Graphical Calculi: Centers, Zeroth Hochschild Homology and Possible Compositions of Induction and Restriction Functors in Various Diagrammatical Algebras. Thesis (Ph.D.), Columbia University, ProQuest LLC (2011)

6. Crane, L., Frenkel, I.B.: Four-dimensional topological quantum field theory, Hopf categories, and the canonical bases. Topology and physics. J. Math. Phys. 35(10), 5136-5154 (1994)

7. Fan, C.K., Green, R.M.: On the affine Temperley-Lieb algebras. J. Lond. Math. Soc. (2) 60(2), 366-380 (1999)

8. Fomin, S., Stanley, R.P.: Schubert polynomials and the nil-Coxeter algebra. Adv. Math. 103(2), 196-207 (1994)

9. Green, R.M.: On 321-avoiding permutations in affine Weyl groups. J. Algebraic Comb. 15(3), 241-252 (2002)

10. Khovanov, M.: Nilcoxeter algebras categorify the Weyl algebra. Commun. Algebra 29(11), 5033-5052 (2001)

11. Kostant, B., Kumar, S.: The nil Hecke ring and cohomology of $G / P$ for a Kac-Moody group $G$. Adv. Math. 62(3), 187-237 (1986)

12. Korff, C., Stroppel, C.: The $\widehat{\mathfrak{s l}(}(n)_{k}-\mathrm{WZNW}$ fusion ring: a combinatorial construction and a realisation as quotient of quantum cohomology. Adv. Math. 225(1), 200-268 (2010)

13. Koenig, S., Xi, C.: Affine cellular algebras. Adv. Math. 229(1), 139-182 (2012)

14. Lam, T.: Affine Stanley symmetric functions. Am. J. Math. 128(6), 1553-1586 (2006)

15. Lam, T.: Schubert polynomials for the affine Grassmannian. J. Am. Math. Soc. 21(1), 259-281 (2008)

16. Lascoux, A., Schützenberger, M.-P.: Fonctorialité des polynômes de Schubert. Invariant theory (Denton, TX, 1986), Contemp. Math., vol. 88, Am. Math. Soc. Providence, RI, pp. 585-598 (1989)

17. Macdonald, I.G., Schubert polynomials. Surveys in combinatorics, 1991 (Guildford, 1991), London Math. Soc. Lecture Note Ser., vol. 166, Cambridge Univ. Press, Cambridge, pp. 73-99 (1991)

18. Meinel, J.: Affine nilTemperley-Lieb Algebras and Generalized Weyl Algebras: Combinatorics and Representation Theory. Dissertation, Bonn (2016)

19. Postnikov, A.: Affine approach to quantum Schubert calculus. Duke Math. J. 128(3), 473-509 (2005)

20. Ridout, D., Saint-Aubin, Y.: Standard modules, induction and the Temperley-Lieb algebra. Adv. Theor. Math. Phys. 18(5), 957-1041 (2014) 